\title{
3 Research Square

\section{Enterococcus Faecium is a Risk Factor for The Outbreak of Anxiety and Depression in Patients With Inflammatory Bowel Disease}

Hyo-Min Jang

Kyung Hee University

Jeon-Kyung Kim

Kyung Hee University

Min-Kyung Joo

Kyung Hee University

Yun-Jung Shin

Kyung Hee University

Kyung-Eon Lee

Kyung Hee University

Joong-Wook Yoo

Kyung Hee University

Chang-Kyun Lee

Kyung Hee University College of Medicine: Kyung Hee University School of Medicine

Hyo-Jong Kim

Kyung Hee University College of Medicine: Kyung Hee University School of Medicine

Dong-Hyun Kim ( $\nabla$ dhkim@khu.ac.kr)

Kyung Hee University https://orcid.org/0000-0003-4783-7900

\section{Research}

Keywords: inflammatory bowel disease, depression, fecal microbiota transplantation, gut microbiota, Enterobacteriaceae, Enterococcaceae, Bifidobacteria

Posted Date: November 13th, 2020

DOI: https://doi.org/10.21203/rs.3.rs-104568/v1

License: (c) (1) This work is licensed under a Creative Commons Attribution 4.0 International License.

Read Full License 


\section{Abstract}

\section{Background}

The gut microbiota closely communicate with the brain through the microbiota-gut-brain axis. The interaction between gut microbiota may regulate the occurrence of neuropsychiatric disorders, including depression. Therefore, we transplanted the fecal microbiota of patients with inflammatory bowel disease (IBD) or their overpopulated gut bacteria into specific-pathogen-free or germ-free mice and examined their effects regarding the occurrence of colitis and anxiety/depression.

\section{Results}

Fecal microbiota transplantations (FMTs) from patients with IBD with $\left(/ \mathrm{D}^{+}\right)$or without depression (/D) caused IBD-like colitis in the transplanted mice: they increased myeloperoxidase activity and NF$\mathrm{KB}^{+} / \mathrm{CD} 11 \mathrm{c}^{+}$cell population in the colon. FMTs from patients with IBD/ $\mathrm{D}^{+}$caused anxiety-/depression-like behaviors and $\mathrm{NF}-\mathrm{KB}^{+} / \mathrm{Iba} 1^{+}$and lipopolysaccharide $(\mathrm{LPS})^{+} / \mathrm{Iba} 1^{+}$cell population and decreased the $\mathrm{BDNF}^{+} / \mathrm{NeuN}^{+}$cell population in the hippocampus. FMTs from patients with IBD/D caused anxiety-like, but not depression-like, behaviors. $a$-/ $\beta$-diversities and composition of microbiota in the feces of patients with IBD (IBD-F) were different from those of healthy-control feces (HC-F). The Enterobacteriaceae and Enterococcaceae populations and fecal lipopolysaccharide levels were higher in IBD-F vs. HC-F. Moreover, the Enterococcaceae population was higher in IBD/D+ $-\mathrm{F}$ vs. IBD/D-F, while the Bifidobacteria population was lower in IBD/D ${ }^{+}-F$. FMT from $\mathrm{HC}$ alleviated the IBD/D+-F-induced anxiety-/depression-like behaviors and colitis in the transplanted mice. Furthermore, it suppressed IBD/D ${ }^{+}-$F-induced Enterococcus sp. population in the feces. Enterobacteriaceae Klebsiella oxytoca, Klebsiella pneumoniae, Escherichia coli, and Cronobacter sakazakii abundant in IBD-F, singly or together, caused depression with colitis in germfree and specific-pathogen-free mice, while Enterococcus faecium abundant in IBD/ $D^{+}-F$ did not cause not anxiety/depression and colitis. However, the combination of Enterobacteriaceae with Enterococcus faecium synergistically deteriorated depression and colitis, while its combination with Bifidobacterium longum attenuated them.

\section{Conclusion}

The interaction between gut microbiota Enterobacteriaceae, Enterococci, and Bifidobacteria may regulate the outbreak of anxiety/depression and IBD through the modulation of NF-KB-involved BDNF expression and gut microbiota. Enterococcus faecium, a probiotic strain, is a risk factor for the outbreak of anxiety/depression in patients with IBD.

\section{Background}

Inflammatory bowel disease (IBD), including ulcerative colitis (UC) and Crohn's disease (CD), is a chronic and recurrent disorder characterized by alternately repeating exacerbations and remissions [1]. Although 
obscure, the aetiology of IBD is thought to be the dysregulation of the mucosal immune system in the gut, resulting in an abnormal inflammatory response to environmental factors such as gut microbiota [2, 3]. In patients with IBD, the prevalence of psychiatric disorders such as anxiety and depression is significantly higher than it is in healthy individuals [4, 5]. Evidence in support of the close connection between IBD and psychiatric disorders stems primarily from animal and human studies [5, 6]. Exposure to an acute psychological stress increases the secretion of the pro-inflammatory cytokines tumor necrosis factor a (TNF-a), interleukin-1 (IL-1) and IL-6 in both the blood and colon mucosa of patients with IBD [7-9]. In the Manitoba IBD Cohort Study population, $80 \%$ of patients with both IBD and anxiety disorder received the diagnosis of anxiety disorder more than 2 years before the IBD diagnosis [10]. Excessive exposure of mice to stressors, such as immobilization and pathogens, stimulates the secretion of adrenal hormones, such as cortisol, and immune cytokines, such as IL-1 $\beta$ and IL- 6 , via the activation of the hypothalamus-pituitary-adrenal (HPA) axis, resulting in the occurrence of colitis and gut dysbiosis accompanied by anxiety, depression, and memory impairment [11-13]. Antidepressant drugs attenuate colitis [14], anti-inflammatory drugs alleviate psychiatric disorders with colitis [9]. These findings suggest that the brain can bidirectionally communicate with the gut through the HPA and gut-brain axes.

The gut microbiota of healthy humans and animals consist of bacteria, viruses, fungi, archaea, and multicellular parasites [15]. Of these, commensal opportunistic pathogens, such as Escherichia coli and Klebsiella oxytoca, produce toxic byproducts, including lipopolysaccharide (LPS) [16, 17]. Their overgrowth by stressors, such as antibiotics and immobilization stress, dysregulate gut immune homeostasis, resulting in the occurrence of gut inflammation through the excessive expression of proinflammatory cytokines $[12,18]$. Excessive exposure to pro-inflammatory cytokines attenuates the expression of tight-junction proteins in the intestine, resulting in a leaky gut, as observed in patients with $\operatorname{IBD}[19,20]$. The induction of leaky gut by gut inflammation accelerates the absorption of bacterial byproducts, such as LPS, into the blood and alters the gut microbiota composition, which is termed dysbiosis [20-22]. Patients with IBD, including UC and CD, exhibit a reduced gut microbial diversity compared with healthy individuals [23-25]. Lepage et al. reported that patients with UC exhibit the increased Actinobacteria and Proteobacteria populations compared with their healthy siblings [26]. In turn, Alam et al. reported that the Proteobacteria and Firmicutes populations were significantly increased in patients with CD compared with healthy individuals, while patients with UC exhibited a decreased Proteobacteria population compared with healthy individuals [23]. The fecal microbiota transplantation (FMT) from patients with IBD causes the colitis in transplanted germ-free mice [27, 28]. The FMT from mice with colitis causes depression with colitis in transplanted specific-pathogen-free mice [12, 29]. The occurrence of colitis and psychiatric disorder triggered by intestinal environmental factors, such as antibiotics and pathogens, can be attenuated by treatment with commensal Lactobacilli and Bifidobacteria $[12,18]$. These findings suggest that the microbiota can communicate with the brain through the microbiota-gut-brain (MGB) axis, and that the interaction between gut microbiota may regulate the occurrence of neuropsychiatric disorders, including depression.

In the present study, we transplanted the fecal microbiota of patients with IBD or their overpopulated gut bacteria into specific-pathogen-free or germ-free mice and examined their effects regarding the 
occurrence of colitis and anxiety/depression.

\section{Results}

\section{Fecal microbiota transplantations from patients with IBD caused anxiety/depression as well as colitis in the transplanted mice}

To understand the role of gut microbiota in the occurrence of IBD and anxiety/depression, we transplanted the fecal microbiota of healthy controls (HCs) or patients with IBD with depression (IBD/D ${ }^{+}$, score $>10$ on HADS-D) or without depression (IBD/D-) into specific-pathogen-free mice and examined their effects regarding the occurrence of colitis and anxiety/depression. The FMTs of patients with IBD/D ${ }^{+}$or $\mathrm{IBD} / \mathrm{D}^{-}$all also significantly increased anxiety-like behaviors in the transplanted mice in the elevated plus maze (EPM), light/dark transition (LDT), and marble burying (MB) tasks (Figure 1a-c). Moreover, depression-like behaviors in the tail suspension test (TST) and forced swimming test (FST) were significantly increased by the FMTs of patients with IBD/D ${ }^{+}$, but not those of patients with IBD/D- (Figure $1 \mathrm{~d}, \mathrm{e})$. The FMTs from patients with IBD also increased NF-KB ${ }^{+} / \mathrm{Iba} 1^{+}$, and $\mathrm{LPS}^{+} / \mathrm{Iba} 1^{+}$, and IL-1 $\mathrm{R}^{+}$cell populations, as well as IL-1 $\beta$ expression in the hippocampus, while the $\mathrm{BDNF}^{+} / \mathrm{NeuN}^{+}$cell population and claudin-5 (a tight-junction protein) were reduced (Figure $1 \mathrm{f}-\mathrm{h}$ ). They also increased corticosterone, IL-1 $\beta$, IL-6, and LPS levels in the blood (Figure 1 $\mathrm{i}-\mathrm{I}$ ). In the present study, each human feces was transplanted into three mice. Therefore, we analyzed their effects by using all mouse data or average values of three mouse data (Figure 1, Supplementary Figure S1). However, the difference between these analyses was not significant. After the segregation of the IBD population into $U C / D^{-}, U C / D^{+}, C D / D^{-}$, and $C D / D^{+}$, the FMTs of patients with $\mathrm{CD} / \mathrm{D}^{+}, \mathrm{CD} / \mathrm{D}^{-}$, and $\mathrm{UC} / \mathrm{D}^{+}$caused depression-like behaviors in mice, while mice with FMT from patients with UC/D` did not show depression-like behaviors (Supplementary Figure S2-S4). The neuroinflammatory markers IL- $1 \beta$ and IL- 6 and the NF-KB ${ }^{+} / \mathrm{CD} 11 \mathrm{c}^{+}$cell population were most potently induced in mice with FMT from patients with $C D / D^{+}$, followed by mice with those from patients

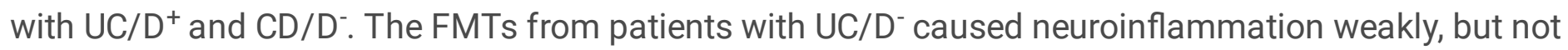
significantly. Moreover, the FMT from HCs did not significantly affect neuroinflammation markers.

The FMTs from patients with IBD/D ${ }^{+}$or IBD/Dª all caused colitis in mice: they significantly induced colon shortening; upregulated myeloperoxidase activity, IL-1 $\beta$ and IL- 6 expression; increased the NF$\mathrm{KB}^{+} / \mathrm{CD} 11 \mathrm{c}^{+}$cell population in the colon (Figure 2a-h, Supplementary Figure S5). In particular, the stenosis was a significant occurrence in mice with $\mathrm{FMTs}$ from IBD/D ${ }^{+}$patients vs. mice with $\mathrm{FMTs}$ from IBD/D` patients. In contrast, the FMTs from HCs did not cause colitis. After segregation of the IBD population into patients with UC patients without depression (UC/D') and with depression $\left(U C / D^{+}\right)$and patients with $C D$ without depression (CD/D) and with depression $\left(C D / D^{+}\right)$, the FMTs of patients with UC and $C D$ all caused colitis in mice, regardless of the presence/absence of depression: they increased colon shortening; upregulated myeloperoxidase activity, IL-1 $\beta$ and IL- 6 expression; increased the NF$\mathrm{KB}^{+} / \mathrm{CD} 11 \mathrm{c}^{+}$cell population in the colon (Supplementary Figure S6-S8). Although the occurrence of IBD- 
like colitis between FMTs of UC and CD patients was not significantly different between the FMTs of patients with $U C$ and $C D$, stenosis was a significant occurrence in mice with FMTs from patients with CD, but not those of patients with UC (Supplementary Figure S7). Their effects analyzed by using average values of three mouse data or all mouse data was not significantly different (Figure 2, Supplementary Figure S5).

\section{Fecal microbiota composition in patients with IBD/D ${ }^{+}$and IBD/D-}

The FMTs from patients with IBD significantly caused colitis and anxiety/depression in transplanted mice compared with those from HCs. Therefore, to understand the role of gut microbiota in depression and colitis, we analyzed the gut microbiota composition of the $\mathrm{HC}$ feces (HC-F), IBD/D ${ }^{+}-\mathrm{F}$ and IBD/D-F using pyrosequencing. The a-diversity estimated operational taxonomic unit (OTU) richness, but not Shannon's diversity index, was lower in the feces of patients with IBD than it was in the feces of HC volunteers (Figure 3A,B). Moreover, the OUT richness was lower in IBD/D+-F vs. IBD/D-F. The $\beta$-diversity and bacterial community were significantly different between IBD/D ${ }^{+}-F$ and IBD/D-F. At the phylum level, the Proteobacteria population was higher in the IBD-F compared with $\mathrm{HC}-\mathrm{F}$, while the Bacteroidetes population was lower in the IBD-F. At the family level, the populations of Enterobacteriaceae, Enterococcaceae, and Lactobacillaceae were higher in the IBD-F compared with HC-F (Figure 3B,C). In particular, the Enterococcaceae and Lactobacillaceae populations were higher in the IBD/D ${ }^{+} \mathrm{F}$ vs. IBD/DF. Among the gut bacteria, the Enterobacterales_f $\left(R^{2}=0.302, p=0.010\right)$, Enterococcus $\left(R^{2}=0.220, p=0.032\right)$, Lactobacillaceae $\left(R^{2}=0.110, p=0.142\right)$, and Pediococcus acidilactici group $\left(R^{2}=0.111, p=0.141\right)$ populations were positively correlated with the depression index in patients with IBD (HADS-D), whereas the Enterobacterales_f $\left(R^{2}=0.344, p=0.005\right)$, Enterobacterales_g $\left(R^{2}=0.344, p=0.005\right)$, Enterobacterales group $\left(R^{2}=0.344, p=0.005\right)$, Bacteroides uniformis $\left(R^{2}=0.346, p=0.005\right)$, and Pediococcus acidilactici group $\left(R^{2}=0.240, p=0.024\right)$ populations were positively correlated with the anxiety index in patients with IBD (HADS-A) (Figure 3D, Supplementary Figure S9). After segregation of the IBD population into HC-F, $U C / D^{-}-F, U C / D^{+}-F, C D / D^{-}-F$, and $C D / D^{+}-F$, the a-diversity (OTU richness) was the lowest in the $C D / D^{+}-F$, whereas those of $U C / D^{-}-F, U C / D^{+}-F$, and $C D / D^{-}-F$ were not significantly different (Supplementary Figure S9S11). The $\beta$-diversity and bacterial community were different between the IBD/D+-F and IBD/D-F. At the phylum level, the Proteobacteria population was the highest in the $C D / D^{-}-F$, followed by $C D / D^{-}-F, C D / D^{+}-F$, $\mathrm{UC} / \mathrm{D}^{+}-\mathrm{F}$ and $\mathrm{UC} / \mathrm{D}^{-}-\mathrm{F}$, while the Bacteroidetes population was the lowest in the $C D / \mathrm{D}^{-}-\mathrm{F}$. At the family level, the populations of Enterobacteriaceae and Enterococcaceae were higher in the UC-F and CD-F than they were in the HC-F, while the Acidaminococcaceae population was lower in the UC-F and CD-F than it was in the HC-F (Supplementary Figure S10). The Bacteroidaceae population was lower in the CD-F than it was in the UC-F.

Next, to confirm the role of live gut bacteria in depression and colitis, we cultured the fecal bacteria of HC$\mathrm{F}$ and IBD-F (IBD/D-F and IBD/D $-F$, respectively) in the Enterobacteriaceae-selective deoxycholate hydrogen sulfate lactose (DHL), and Enterococcus-selective m-Enterococcus (En), and Bifidobacteriaselective blood liver (BL) agar plates (Figure 3E). Klebsiella sp. (Klebsiella oxytoca and Klebsiella 
pneumoniae) and Escherichia coli and Cronobacter sakazakii populations (in DHL agar plates) and Enterococcus sp. and Pediococcus acidilactici populations (in En agar plates) were highly detected in the IBD-F compared with HC-F. The Cronobacter sakazakii and Pediococcus acidolacticipopulations were not significantly different among $\mathrm{HC}-\mathrm{F}, \mathrm{IBD} / \mathrm{D}^{-}-\mathrm{F}$ and IBD $/ \mathrm{D}^{+}-\mathrm{F}$. However, the Bifdiobacterium sp. population was most highly detected in the HC-F, followed by the feces of patients with UC/D-F, UC/D+-F, CD/D-F, and $\mathrm{CD} / \mathrm{D}^{+}-\mathrm{F}$ (Figure S11C). The populations of Escherichia coli, Klebsiella oxytoca,Klebsiella pneumoniae, Cronobacter sakazakii, and Enterococcus faecium in the HC-F and IBD-F were also analyzed by using quantitative real-time polymerase reaction (qPCR) (Figure 3F). The populations of Escherichia coli, Klebsiella pneumonae,Klebsiella oxytoca, and Enterococcus sp. were more highly detected in the IBD$\mathrm{F}$ compared with $\mathrm{HC}-\mathrm{F}$, while the Bifidobacteria population was lower.

The population of Enterococcus sp. was higher in the IBD/D $-\mathrm{F}$ vs. IBD/D-F, while the Pediococcus acidolactici population was not significantly different among HC-F, IBD/D-F and IBD/D+-F. The populations of Escherichia coli, Klebsiella pneumonae,Klebsiella oxytoca, and Cronobacter sakazakii were not significantly different between the feces of UC and CD patients. However, Bifidobacterium sp. was detected at their highest levels in $H C-F$, followed by $U C / D^{-}-F, U C / D^{+}-F$, and $C D / D^{+}-F / C D / D^{-}-F$ (Supplementary Figure S9-S11).

Next, we analyzed the content of bacterial LPS in the feces of the HC-F, IBD/D-F, and IBD/D $-F$ (Figure 3G). The fecal LPS content was higher in the IBD-F than it was in the HC-F, while the differences between $\mathrm{IBD} / \mathrm{D}^{-}-\mathrm{F}$ and IBD $/ \mathrm{D}^{+}-\mathrm{F}$ and between $\mathrm{UC}-\mathrm{F}$ and $\mathrm{CD}-\mathrm{F}$ were not significant.

\section{Fecal microbiota composition in mice transplanted with IBD/D+-F or IBD/D-F}

IBD-F transplantation significantly caused colitis and anxiety/depression in transplanted mice compared with HCs. Therefore, we analyzed the gut microbiota composition of the feces of mice transplanted with $\mathrm{IBD} / \mathrm{D}^{+}-\mathrm{F}$ or IBD/D-F using pyrosequencing. The a-diversity (OUT richness), but not Shannon's diversity index, was significantly higher in IBD-F than in HC-F (Figure 4A-C). The $\beta$-diversity was also significantly different between the gut microbiota compositions of mice transplanted with HC-F and IBD-F (UC/D-F, $U C / D^{+}-F, C D / D^{-}-F$ or $\left.C D^{-}-F\right)$. Compared with the bacterial composition at the phylum level, the Proteobacteria population was higher in the feces of mice transplanted with IBD-F than in those of mice transplanted with HC-F (Figure 4C, Supplementary Figure S12,S13). At the family level, the Enterococcaceae, Bacteroidaceae, and Prevotellaceae populations were increased by the FMT of IBD-F vs. HC-F. Furthermore, the Enterococcaceae populations were higher in the IBD/D+ $-F$ vs. IBD/D-F (Figure 4C). Among the gut bacteria, the Enterobacteriaceae $\left(R^{2}=0.087, p=0.152\right)$, Enterococcaceae $\left(R^{2}=0.371\right.$, $\mathrm{p}=0.001)$, Lactobacillaceae $\left(R^{2}=0.227, p=0.016\right)$, Prevotellaceae $\left(R^{2}=0.450, p<0.001\right)$, Bacteroides oleiciplenus group $\left(R^{2}=0.456, p<0.001\right)$, and Enterococcus faecium group $\left(R^{2}=0.371, p=0.001\right)$ populations were positively correlated with the depression index of patients with IBD (HADS-D), while the Dexulfovibrionaceae $\left(\mathrm{R}^{2}=0.352, \mathrm{p}=0.002\right)$, Enterococcaceae $\left(\mathrm{R}^{2}=0.268, \mathrm{p}=0.008\right)$, Prevotellaceae $\left(R^{2}=0.280, p=0.007\right)$, Bacteroides oleiciplenus group $\left(R^{2}=0.180, p=0.034\right)$, Enterococcus faceium group 
$\left(R^{2}=0.268, p=0.008\right)$, Lactobacillus_uc $\left(R^{2}=0.099, p=0.126\right)$ populations were positively correlated with the anxiety index of patients with IBD (HADS-A) (Figure 4D, Supplementary Figure S13). The FMTs from patients with IBD increased the population of Enterococcus faecium, but not Pediococcus acidilactoci, which is assessed by qPCR, and LPS level compared with those from HC while the differences between IBD/D-F and IBD/D $-F$ and between UC-F and CD-F were not significant (Figure 4E,F, Supplementary Figure S13-S15).

\section{Klebsiella oxytoca caused colitis and depression in germ-free and specific pathogen-free mice}

To understand the difference in the occurrence of colitis and depression caused by Enterobacteriaceae between in germ-free and specific-pathogen-free mice, we orally gavaged Klebsiella oxytoca into germfree and specific-pathogen-free mice and examined its effects on the occurrence of colitis and depression (Figure 5). Klebsiella oxytoca caused colitis and anxiety/depression in the specific-pathogen-free mice. Oral gavage of Klebsiella oxytoca at a dose of $1 \times 10^{8} \mathrm{CFU} / \mathrm{mouse} /$ day into four mice strongly increased $\mathrm{NF}-\mathrm{KB}^{+} / \mathrm{Iba} 1^{+}$and $\mathrm{LPS}^{+} / \mathrm{Iba} 1^{+}$cell populations, upregulated IL-1 $\beta$ expression and decreased the $\mathrm{BDNF}^{+} / \mathrm{NeuN}^{+}$cell population in the hippocampus; whereas it upregulated IL-1 $\beta$ expression, myeloperoxidase activity, and the NF- $\mathrm{KB}^{+} / \mathrm{CD} 11 \mathrm{c}^{+}$cell population in the colon (Figure $5 \mathrm{~A}$ ).

Oral gavage of Klebsiella oxytoca also at a dose of $1 \times 10^{7} \mathrm{CFU} /$ mouse/day caused colitis and anxiety/depression in germ-free mice. The incidence of depression and colitis in germ-free mice by Klebsiella oxytoca at a dose of $1 \times 10^{7} \mathrm{CFU} /$ mouse/day was similar to one treated at doses of $1 \times 10^{8}$ $\mathrm{CFU} /$ mouse/day in specific-pathogen-free mice (Figure 5B).

\section{Enterobacteriaceae, Pediococcus acidilactici, Enterococcus faecium, and Bifidobacterium longum interactively regulated the occurrence of colitis and depression in mice}

We examined the effects of the gut bacteria Klebsiella oxytoca, Klebsiella pneumoniae, Escherichia coli, Cronobacter sakazakii,Enterococcus faecium,Pediococcus acidilactici and Bifidobacterium longum on the occurrence of colitis and depression in specific-pathogen-free mice. Of these bacteria, Klebsiella pneumoniae exhibited the highest lethal toxicity in specific-pathogen-free mice, followed by Klebsiella oxytoca and Escherichia coli. Oral gavage of Klebsiella pneumoniae at doses of $1^{\prime} 10^{7}$ and $^{3} 1^{\prime} 10^{8}$ $\mathrm{CFU} /$ mouse/day was lethal in $0 \%$ and $100 \%$ of mice within 5 days, respectively (Figure $6 \mathrm{~A}$ ). Oral gavage of Klebsiella oxytoca at doses of $1^{\prime} 10^{8}$ and $1^{\prime} 10^{9} \mathrm{CFU} / \mathrm{mouse} /$ day was lethal in $0 \%$ and $50 \%$ of mice within 5 days, respectively. Oral gavage of Escherichia coli, Cronobacter sakazakii,Enterococcus faecium,Pediococcus acidilactici or Bifidobacterium longum at a dose of $1^{\prime} 10^{9} \mathrm{CFU} / \mathrm{mouse} /$ day did not kill any mice. Oral gavage of Klebsiella pneumoniae at doses of ${ }^{3} 1^{\prime} 10^{6} \mathrm{CFU} / \mathrm{mouse} /$ day, Klebsiella oxytoca at doses of ${ }^{3} 1^{\prime} 10^{7} \mathrm{CFU} / \mathrm{mouse} /$ day, Escherichia coli at doses of ${ }^{3} 1^{\prime} 10^{7} \mathrm{CFU} / \mathrm{mouse} /$ day and Cronobacter sakazakii at a dose of $1^{\prime} 10^{9} \mathrm{CFU} /$ mouse/day caused colitis in mice: they increased the stenosis score, myeloperoxidase activity, IL-1 $\beta$ and IL- 6 expression, and the NF-KB ${ }^{+} / \mathrm{CD}_{11} \mathrm{c}^{+}$cell 
population in the colon while claudin-1 and occludin were decreased (Figure 6, Supplementary Figure S16). They also increased corticosterone, IL-1 1 , IL-6, and LPS levels in the blood, as well as LPS level in the feces. Moreover, they caused depression in mice: they increased anxiety/depression-like behaviors, IL$1 \beta$ expression, and the NF-KB ${ }^{+} / \mathrm{Iba}^{+}{ }^{+}, \mathrm{LPS}^{+} / \mathrm{Iba}^{+}$, and $\mathrm{IL}-1 \mathrm{R}^{+}$cell populations in the hippocampus while the $\mathrm{BDNF}^{+} / \mathrm{NeuN}^{+}$cell population and claudin- 5 expression were decreased. However, oral gavage of Enterococcus faecium, Pediococcus acidilactici, or Bifidobacterium longum at a dose of $1^{\prime} 10^{9}$ $\mathrm{CFU} /$ mouse/day did not significantly cause colitis and depression in mice.

The combination of two bacteria among Klebsiella oxytoca, Klebsiella pneumoniae, Escherichia coli, and Cronobacter sakazakii, these combinations synergistically or additively increased the mortality and the occurrence of depression and colitis in mice (Figure 7, Supplementary Figure S17). Of these combinations, that of Klebsiella pneumoniae with Klebsiella oxytoca (KpKo, 1:1) increased mortality in mice most strongly, followed by Klebsiella pneumoniae with Escherichia coli (KpEc, 1:1)/Klebsiella pneumoniae with Cronobacter sakazakii (KpCs, 1:1), and Klebsiella oxytoca with Escherichia coli (KoEc, 1:1)/Klebsiella oxytoca with Cronobacter sakazakii (KoCs, 1:1) (Figure 7). KpKo at doses of ${ }^{3} 1^{\prime} 10^{8}$ $\mathrm{CFU} /$ mouse/day and $\mathrm{KpCs}$ and $\mathrm{KpEc}$ at a dose of $1 ' 10^{9} \mathrm{CFU} / \mathrm{mouse} /$ day killed all mice within 5 days. KoKp at a dose of $1^{\prime} 10^{6}$ and $1^{\prime} 10^{7} \mathrm{CFU} /$ mouse/day killed $33.3 \%$ and $50 \%$ of mice, respectively. KpEc at a dose of $1^{\prime} 10^{8} \mathrm{CFU} / \mathrm{mouse} /$ day killed $83.3 \%$ of mice. KpCs at doses of $1^{\prime} 10^{7}$ and $1^{\prime} 10^{8}$ $\mathrm{CFU} /$ mouse/day killed $33.3 \%$ and $66.7 \%$ of mice, respectively. KoEc and KoCs at a dose of $1^{\prime} 10^{9}$ killed $50 \%$ of mice. Other combinations did not kill mice. Oral gavage of $\mathrm{KpPa}$ also caused depression and colitis most strongly, followed by KpEc/KpCs, KoEc and EcCs (Figures 7).

Next, we examined the combined effects of Enterococcus faecium and Bifidiobacterium longum on the mortality and the occurrence of depression and colitis caused by Klebsiella oxytoca, Klebsiella pneumoniae, and Escherichia coli in mice (Figure 8, Supplementary Figure S18). The combinations of Enterococcus faecium with Klebsiella oxytoca (KoEf, 1:1), Klebsiella pneumoniae (KpEf, 1:1), and Escherichia coli (EcEf, 1:1) did not affect the mortality of Klebsiella oxytoca, Klebsiella pneumoniae, and Escherichia coli in mice, respectively (Figures 7a, 8a). However, Enterococcus faecium synergistically increased the occurrence of depression and colitis of Klebsiella oxytoca, Klebsiella pneumoniae, and Escherichia coli (Figure 8b-h, Supplementary S17). However, the combination with Bifidobacterium longum significantly suppressed the mortality and occurrence of depression and colitis causable by Klebsiella oxytoca, Klebsiella pneumoniae, or Escherichia coli. Furthermore, its combination with Bifidobacterium longum suppressed the increase in the stenosis score and the NF-kB ${ }^{+} / \mathrm{CD} 11 \mathrm{c}^{+}$cell population in the colon, LPS levels in the blood and feces, and the NF-KB $/ / \mathrm{Iba} 1^{+}$and IL-1 $\mathrm{R}^{+}$cell populations in the hippocampus; it also reduced the $\mathrm{BDNF}^{+} / \mathrm{NeuN}^{+}$cell population and claudin- 5 expression in the hippocampus.

\section{FMT from $\mathrm{HC}$ alleviated IBD/D+-F-induced depression and colitis in the transplanted mice}


To confirm the role of gut microbiota in the occurrence of IBD and anxiety/depression, we prepared mice with IBD/D+-F- or IBD/D-F-transplanted depression and colitis and transplanted the fecal microbiota of HCs (Figure 9, Supplement Figure S19). The FMTs from patients with IBD/D ${ }^{+}$all caused anxiety-/depression-like behaviors and colitis. The FMTs from patients with IBD/D caused anxiety-like behaviors, but not depression-like behaviors. However, the FMT from HC suppressed the IBD/D+-Finduced anxiety-like behaviors in the EPM, MB, and LDT tasks, depression-like behaviors in the TST and FST, IL-6 expression in the hippocampus, and corticosterone and IL-6 levels in the blood, resulting in the amelioration of anxiety/depression. The FMT from $\mathrm{HC}$ also suppressed colon shortening and reduced myeloperoxidase activity, and IL- 6 expression in the colon of mice with the IBD/D + -F-induced depression, resulting in the alleviation of colitis. The FMT form $\mathrm{HC}$ also alleviated the IBD/D-F-induced anxiety-like behaviors in the EPM, MB, and LDT tasks, IL- 6 expression in the hippocampus, corticosterone, LPS, and IL-6 levels in the blood, and colon shortening, myeloperoxidase activity, and IL- 6 expression in the colon. Furthermore, the FMT from $\mathrm{HC}$ significantly reduced the IBD/D+-F-induced Enterococcus sp. population and bacterial LPS production in the feces. However, the FMT from HC alleviated anxiety, but not depression, and colitis in IBD/D-F-transplanted mice, while the Enterococcus sp. population was not affected.

\section{Discussion}

Gut microbiota is closely involved in the occurrence of psychiatric disorders through the activation of the MGB axis [30]. Many studies have focused on the role of gut microbiota in the outbreak of neuropsychiatric disorders [30-32]. Klebsiella and Lachnospira, which belong to the Firmicutes phylum, are higher, while the Escherichia and Bifidobacterium populations are lower, in the feces of patients with depression vs. the feces of healthy individuals [33]. The populations of Alistipes (Bacteroidetes), and Enterobacteriaceae are over-represented in patients with depression [33]. The induction of depression by stressors triggers colitis and increases the gut Proteobacteria population and bacterial LPS production in mice $[12,18]$. The FMT from stress-stimulated mice also causes colitis with depression in the transplanted mice [12]. Several studies have demonstrated that the high prevalence of commensal gut Proteobacteria including Enterobacteriaceae is closely associated with IBD, including UC and CD [34], overexpression of gut bacterial LPS. However, to date, none of these species/strains were convincingly shown to be associated with the occurrence of IBD and depression.

In the present study, the FMTs from patients with IBD caused colitis and depression in the transplanted mice: they induced the NF-KB ${ }^{+} / \mathrm{Iba}^{+}{ }^{+} \mathrm{LPS}^{+} / \mathrm{Iba} 1^{+}$and IL- $1 \mathrm{R}^{+}$cell populations and IL-1 $\beta$ and IL- 6 expression in the hippocampus and myeloperoxidase activity and IL-1 $\beta$ and IL- 6 expression in the colon. After the segregation of patients with IBD/D and IBD/D ${ }^{+}$, the FMTs from patients with IBD/D- or IBD/D ${ }^{+}$ all caused colitis and anxiety in the transplanted mice, while the FMTs from patients with IBD/ $\mathrm{D}^{+}$, but not IBD/D', caused depression. They induced myeloperoxidase activity and IL-1 $\beta$ and IL- 6 expression in the colon. The FMTs from patients with IBD/D increased the stenosis score more potently than did the FMTs 
of patients with IBD/D'. However, after the segregation of patients with $U C / D^{-}, U C / D^{+}, C D / D^{-}$, and $C D / D^{+}$, the FMTs from patients with $\mathrm{UC} / \mathrm{D}^{+}, \mathrm{CD} / \mathrm{D}^{-}$or $\mathrm{CD} / \mathrm{D}^{+}$caused colitis with depression in the transplanted mice: they increased the NF-KB ${ }^{+} / \mathrm{Iba}^{+}, \mathrm{LPS}^{+} / \mathrm{Iba}^{+}$and IL-1 $\mathrm{R}^{+}$cell populations and upregulated IL- $1 \beta$ expression in the hippocampus and increased the NF-KB ${ }^{+} / \mathrm{CD} 11 \mathrm{c}^{+}$cell population in the colon. The FMTs from patients with UC/D caused colitis with anxiety, but not depression. Interestingly, the occurrence of colon stenosis was different between the FMTs from patients with UC and CD, as it was observed at a greater extent in mice transplanted with the feces of CD patients than in those transplanted with the feces of patients with UC as patients with IBD [35]. The occurrence of colon stenosis was not significantly different between FMTs of $C D / D^{-}$and $C D / D^{+}$. These results suggest that the gut microbiota consists of IBD-inducing, -suppressing, and -irrelevant microbes and that some IBD-inducing microbes may cause anxiety with or without depression. Moreover, the abundance of depression-inducing microbes may be higher in the gut microbiota of patients with IBD/D+ than those of patients with IBD/D'.

We also found that the gut microbiota composition of IBD-F was significantly different from that of $\mathrm{HC}$ feces: the $\alpha-/ \beta$-diversities of IBD-F were lower than those of HC-F, and the $\beta$-diversity was different between IBD-F and HC-F, as reported previously $[34,36,37]$. Although the a-diversity was not significantly different between IBD/D-F and IBD/D+-F, the $\beta$-diversity was different. IBD-F exhibited a higher abundance of the Proteobacteria population compared with $\mathrm{HC}-\mathrm{F}$, while the Bacteroidetes population was lower in IBD-F. In particular, IBD/D ${ }^{+}-\mathrm{F}$ exhibited a higher abundance of Enterobacteriaceae, Enterococcaceae, and Lactobacillaceae compared with IBD/D-F, while the Acidaminococcaceae population decreased in IBD-F compared with HC-F. Assessed by the selective medium culture and QPCR, IBD-F exhibited a significantly higher abundance of Escherichia coli, Klebsiella oxytoca, Klebsiella pneumoniae and Enterococcus faecium and a lower abundance of Bifidobacterium sp. (including Bifidobacterium longum). Among IBDF, UC/D-F exhibited a higher abundance of Bifidobacterium sp., followed by UC/D ${ }^{+}-\mathrm{F}$ and $\mathrm{CD} / \mathrm{D}^{-}-\mathrm{F} / \mathrm{CD}^{-} \mathrm{D}^{+}-$ F. The population of Enterococcus sp. was higher in IBD/D ${ }^{+}-F$ vs. IBD/D-F. Moreover, the gut bacterial LPS level was higher in the IBD-F than it was in the HC-F. However, the differences in these bacterial population between IBD/D-F and IBD/D+-F and between UC-F and CD-F were not significant. Among these gut bacteria, the Enterobacterales_f and Enterococcus populations were positively correlated with the depression index in patients with IBD (HADS-D) and the Enterobacterales_f, Enterobacterales_g, Alistipes, and Enterobacterales group populations were positively correlated with the anxiety index in patients with IBD (HADS-A). These results suggest that the IBD with depression may be induced in the intestine by Enterobacteriaceae, Enterococcaceae, and Lactobacillaceae and suppressed by Bifidobacterium sp.

The fecal microbiota compositions of mice transplanted with HC-F or IBD-F partially matched those of patients with IBD. The Proteobacteria population, including Enterococcaceae, was increased in the feces of mice transplanted with IBD-F than it was in those transplanted with HC-F, similar to those of IBD-F itself. The Enterococcaceae, Lactobacilliaceae, and Enterobacteriaceae populations were positively correlated with the anxiety/depression index of patients with IBD (HADS-A and HADS-D). However, the 
Bacteroidaceae, Prevotellaceae, and Helicobacteraceae populations were increased in the feces of mice transplanted with IBD-F compared with mice transplanted with HC-F. These results support the suggestion that the gut microbiota of IBD-F comprises IBD-inducing microbiota, such as Enterobacteriaceae and Enterococacceae, which may be transient and attachable in the gastrointestinal tract, respectively.

Among the FMTs from IBD patients, the FMT from patients with $C D / D^{+}$induced the depression in the transplanted mice most strongly, followed by patients with $U C / D^{+}, C D / D^{-}$, and $U C / D^{-}$. Among IBD-F and

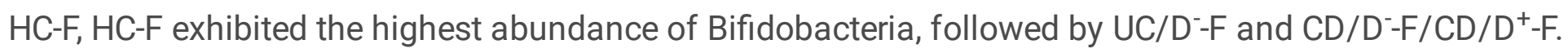
However, gut microbiota of mice transplanted with HF-F or UC/D-F did not be significantly increased. These results suggest that Bifidobacteria may protect against the occurrence of depression caused by Enterobacteriaceae in mice. Moreover, the content of LPS was higher in the IBD-F than in the HC-F, while the differences between IBD/D-F and IBD/D ${ }^{+}-F$ and between UC-F and CD-F were not significant. In addition, the fecal and blood LPS levels were higher in IBD-F-gavaged mice than they were in HC-Fgavaged mice. The FMTs from IBD patients significantly suppressed tight-junction protein expression in the colon and hippocampus. They also suppressed $\mathrm{BDNF}^{+} / \mathrm{NeuN}^{+}$cell population in the hippocampus, while the NF-KB ${ }^{+} / \mathrm{Iba}^{+}{ }^{+}, \mathrm{LPS}^{+} / \mathrm{Iba} 1^{+}$and IL-1 $\mathrm{R}^{+}$cell populations and IL-1 $\beta$ and IL- 6 expression were increased. Among the IBD-F, the levels of IBD markers such as IL-1 $\beta$, IL-6, LPS, and NF-KB ${ }^{+} / \mathrm{Iba}^{+}$cells were induced most strongly by $C D / D^{+}-F$, followed by $U C / D^{+} / C D / D^{-}$and $U C / D^{-}$. Jang et al. reported that the overexpression of fecal LPS after exposure to Escherichia coli significantly suppressed the expression of tight-junction proteins in the brain and colon [12]. Kim et al. reported that Escherichia coli treatment increased the NF-KB ${ }^{+} / \mathrm{Iba} 1^{+}$and $\mathrm{LPS}^{+} / \mathrm{Iba} 1^{+}$cell populations in the hippocampus and LPS levels in the blood and feces and decreased the $\mathrm{BDNF}^{+} / \mathrm{NeuN}^{+}$cell population and tight-junction protein expression, resulting in the occurrence of depression [29]. Lee et al. also reported that oral gavage of Escherichia coli and LPS caused colitis and neuroinflammation in mice through the suppression of tight-junction proteins [38]. Koo et al. showed that the inhibition of IL-1 $\beta$ expression by pre-treatment with an IL-1R antagonist mitigated depression [39]. Exposure of conventional mice to stressors such as high-fat diet and ampicillin caused gut dysbiosis: they increased the Proteobacteria population and gut bacterial LPS production, caused gastrointestinal inflammation and suppressed tight-junction protein expression, resulting in the occurrence of leaky gut $[12,40,41]$. In turn, IBD patients suffer from leaky gut [42], a condition that accelerates the translocation of gut bacteria and their by-products, such as LPS, across the intestinal mucosa and directly the stimulate systemic inflammatory response, resulting in the neuroinflammation in the hippocampus $[43,44]$. These results suggest that the FMT from patients with IBD, except patients with $\mathrm{UC} / \mathrm{D}^{-}$, can significantly suppress the NF-KB-mediated BDNF expression in the brain through the overproduction of bacterial LPS induced by gut dysbiosis, resulting in the outbreak of depression.

In the present study, Enterobacteriaceae including Escherichia coli, Klebsiella pneumoniae, Klebsiella oxytoca and Cronobacter sakazakii, which were isolated from the IBD-F, significantly caused anxiety/depression with colitis in specific-pathogen-free mice. However, Bifidobacterium longum, isolated from HC-F, and Enterococcus faecium and Pediococcus acidilactici, isolated from IBD-F, did not cause 
colitis and anxiety/depression in mice. Among these bacteria, Klebsiella pneumoniae caused anxiety/depression and colitis most potently, followed by Klebsiella oxytoca, Escherichia coli, and Cronobacter sakazakii. Klebsiella pneumoniae yielded the highest level of mortality in mice, followed by Klebsiella oxytoca. The remaining bacteria did not cause death in mice. Klebsiella oxytoca isolated from the feces of patients with IBD also strongly caused colitis and depression in mice, as reported previously [18]. The occurrence of depression after exposure to Klebsiella oxytoca at a dose of $1 \times 10^{7}$ $\mathrm{CFU} /$ mouse/day was more severe in germ-free mice than in specific pathogen-free mice. The occurrence of depression by Klebsiella oxytoca at a dose of $1 \times 10^{7} \mathrm{CFU} /$ mouse/day in germ-free mice was similar to that detected in specific-pathogen-free mice at a dose of $1 \times 10^{8} \mathrm{CFU} /$ mouse/day. These results suggest that, of gut commensal microbiota, Enterobacteriaceae are a risk factor for the occurrence of anxiety/depression with colitis. Further research is necessary to understand the toxic substances of Klebsiella sp.

The combination of Escherichia coli, Klebsiella oxytoca, and Klebsiella pneumoniae together or with Enterococcus faecium, which is known as a probiotic strain $[45,46]$, synergistically or additively increased the mortality and occurrence of colitis and depression caused by parenteral bacteria. Of these combinations, KpEf most strongly caused depression and colitis, followed by KoEf and EcEf. They induced the NF-KB ${ }^{+} / \mathrm{Iba} 1^{+}, \mathrm{LPS}^{+} / \mathrm{Iba} 1^{+}$and IL-1 $\mathrm{R}^{+}$cell populations and IL- $1 \beta$ and IL- 6 expression in the hippocampus and myeloperoxidase activity and IL-1 $\beta$ and IL-6 expression in the colon while the BDNF expression was suppressed in the hippocampus. They also increased LPS levels in the blood and feces and suppressed tight-junction protein expression in the colon and brain. However, when combined with Bifidobacterium longum, which is also known as a probiotic strain [47,48], it significantly suppressed the occurrence of depression and colitis caused by Enterobacteriaceae: it reduced the NF-KB ${ }^{+} / \mathrm{lba}^{+}$, $\mathrm{LPS}^{+} / \mathrm{Iba} 1^{+}$and IL-1R ${ }^{+}$cell populations and IL-1 $\beta$ and IL- 6 expression in the hippocampus and myeloperoxidase activity and IL-1 $\beta$ and IL- 6 expression in the colon and increased the BDNF expression in the hippocampus. The simultaneous treatment of Bifidobacterium longum with Enterobacteriaceae also suppressed LPS levels in the blood and feces and induced tight-junction protein expression in the colon and brain. The FMTs from patients with IBD/D+ caused anxiety/depression, colitis, and gut dysbiosis, such as the increase in the Enterococcus sp. population, in the transplanted mice. However, the microbiota transplantation from $\mathrm{HC}-\mathrm{F}$, which had the abundance of the Bifidobacteria population compared with IBD-F, alleviated the IBD/D+-induced anxiety/depression and colitis: it suppressed the IL$1 \beta$ and IL-6 expression in the hippocampus, LPS level in the feces and blood, and myeloperoxidase activity and IL-1 $\beta$ and IL-6 expression in the colon. Furthermore, it reduced the Enterococcus sp. population in the gut microbiota. Khan et al. reported that the populations of Bifidobacteria and Lactobacilli were higher in the IBD-F than in the HC-F [49]. Barandouzi et al. reported that the population of Bifidobacteriaceae, but not Bifidobacterium sp., was higher in the gut microbiota of patients with depression than in those of HCs [50]. Cheung et al. reported that patients with depression exhibited a lower abundance of Bifidobacteria and a higher abundance of Proteobacteria including Enterococcaceae compared with those of in the HCs [51]. These results suggest that, among the gut probiotic bacteria, Enterococcus faecium belonging to Enterococcaceae (Lactobacillales) can accelerate the occurrence of 
colitis and depression caused by Enterobacteriaceae through the induction of gut bacterial LPS production and the suppression of tight junction protein expression, while Bifidobacterium longum can suppress the occurrence of colitis and depression caused by Enterobacteriaceae through the suppression of gut bacterial LPS production and the induction of tight-junction protein expression. Furthermore, Enterococcus faecium deteriorated the suppression of NF-KB-mediated BDNF expression in the brain by Enterobacteriaceae while Bifidobacterium longum alleviated Enterobacteriaceaeinduced BDNF expression. These results suggest that Enterococcus faecium can accelerate the occurrence of depression by Enterobacteriaceae through the regulation of the gut microbiota-mediated NF-KB activation and BDNF expression whereas Bifdiobacterium longum can alleviate the depression. Nevertheless, further research is necessary to elucidate why Enterococcus faecium increases the mortality caused by Enterobacteriaceae including Klebsiella sp.

\section{Conclusion}

FMTs from patients with IBD/D ${ }^{+}$caused colitis and anxiety/depression-like behaviors, while FMTs from IBD/D` patients caused colitis and anxiety-like, but not depression-like, behaviors. IBD-F exhibited a higher abundance of Enterococcaceae, Lactobacillaceae and Enterobacteriaceae populations in IBD-F vs. HC-F. The Enterococcaceae and Lactobacillaceae populations were remarkably higher in IBD/D ${ }^{+}-\mathrm{F}$ vs. IBD/D-F. Among Enterobacteriaceae, the Enterobacteriaceae Klebsiella oxytoca, Klebsiella pneumoniae, Escherichia coli, and Cronobacter sakazakii, singly or together, caused depression with colitis. The combination of Enterobacteriaceae with Enterococcus faecium synergistically deteriorated depression and colitis, while the combination of Enterobacteriaceae with Bifidobacterium longum attenuated them. Therefore, the interaction between gut microbiota Enterobacteriaceae, Enterococci, and Bifidobacteria may regulate the outbreak of anxiety, depression, and IBD through the modulation of NF-KB-involved BDNF expression and gut microbiota. Enterococcus faecium is a risk factor for the outbreak of anxiety and depression in patients with IBD.

\section{Methods}

\section{Volunteers}

Volunteers, consisting of HCs (average age, $38.2 \pm 2.2$ years) and patients with IBD/D $/ \mathrm{D}^{+}$(average age, 46.4 \pm 15.3 years) and IBD/D' (average age, $36.0 \pm 12.6$ years), were recruited from Kyung Hee University (Seoul, Korea) (Supplement Table S1). HCs were enrolled if antibacterial medications had not been administered within 3 month before the stool collection. All patients with IBD enrolled in the study were $>13$ years of age at the diagnosis of IBD, and all diagnoses were confirmed by previously established international criteria based on clinical, endoscopic, histopathological, and radiological findings [52]. The study protocol and consent forms for the stool collection were approved by the Committee for the Care and Use of Clinical Study of the Medical School of Kyung Hee University (IRB File No., KHUH 2018-03- 
006-018 and KHUH 2018-12-004-003). All experiments were conducted in compliance with the principles of the Declaration of Helsinki and the Korean Good Clinical Practice guidelines.

\section{Animals}

SPF C57BL/ 6 mice (male, 6 weeks old, 19-21 g) were purchased from Koatech Inc. (Seoul, Korea). Mice were maintained in wire cages (three mice per cage) with the $5-\mathrm{cm}$ raised wire floor, which was designed to prohibit mice for feeding the feces, under a ventilated condition (three mice/cage, $20^{\circ} \mathrm{C}-22^{\bullet} \mathrm{C}, 50 \% \pm$ 10\% humidity, and 12-h/12-h light/dark cycle in AAALAC-accredited specific pathogen free facility) and fed standard laboratory chow and water ad libitum. Germ-free C57BL/6J mice (male, 18-21 g, 5 weeks old) were purchased from Clea Japan Inc. (Tokyo, Japan). The mouse breeding protocol is described in the Supplementary Information. Germ-free mice were housed in flexible film plastic isolators. All conditions were kept sterile in accordance with The Guidelines for Laboratory Germ-free Animals Care and Usage. Mice were used in the experiments after acclimation for 1 week. All animal experiments were approved by the Institutional Animal Care and Use Committee of Kyung Hee University [IACUC No., KUASP(SE)-18-045, KUASP(SE)-19-290, and KHSASP-20-078] and were performed according to the NIH, AAALAC International, and University Guide for Laboratory Animals Care and Usage.

\section{Culture of gut bacteria}

The fresh feces $(0.5 \mathrm{~g})$ of IBD patients and HCs were immediately collected, immediately suspended in $4.5 \mathrm{~mL}$ of general anaerobic medium (GAM, Nissui Pharmaceutical Inc., Tokyo, Japan) broth on ice, inoculated onto BL and DHL agar plates (Nissui Pharmaceutical Inc.), and cultured aerobically (for DHL agar plates) at $37^{\circ} \mathrm{C}$ for 1 day or anaerobically (for BL and En agar plates) at $37^{\circ} \mathrm{C}$ for 3 days [12]. The colonies grown in agar plates were inoculated into GAM semi-solid media. These bacteria were identified using Gram staining, 16S rRNA gene sequencing, and API kits, as previously reported $[12,18]$. For in vitro and in vivo experiments, gut bacteria including Klebsiella oxytoca, Klebsiella pneumoniae, Escherichia coli, Cronobacter sakazakii, Pediococcus acidilactici,Enterococcus faecium and Bifidobacterium longum were anaerobically cultured in the GAM broth at $37^{\circ} \mathrm{C}(0.8-1.0$ at $600 \mathrm{~nm})[12,18]$. Cultured bacteria were collected by centrifugation for $20 \mathrm{~min}$ at $5000 \mathrm{~g}$, and washed twice with saline. The collected cells $(1 \times$ $10^{10} \mathrm{CFU} / \mathrm{mL}$ ) were suspended in saline.

\section{Treatment with fecal microbiota suspension or gut bacteria in mice}

The feces of IBD patients or HCs were freshly collected, immediately $(<2 \mathrm{~h})$ suspended in saline, filtrated through sterilized gauze and centrifuged at $500 \mathrm{~g}$ at $4^{\circ} \mathrm{C}$ for $5 \mathrm{~min}$. The supernatant fraction $(10 \mathrm{mg}$ [wet weight] $/ \mathrm{kg} /$ day) was used for bacterial isolation and in vivo experiments (Supplementary Figure S20).

To understand the effects of gut microbiota on the occurrence of depression and colitis, each supernatant fraction (10 mg [wet weight] $/ \mathrm{kg} /$ day) and each gut bacterium $\left(1 \times 10^{6}, 1 \times 10^{7}, 1 \times 10^{8}\right.$ or $1 \times$ $10^{9} \mathrm{CFU} / \mathrm{mouse} /$ day, suspended in saline) isolated in the present study was orally gavaged into three and six mice once a day for 5 days, respectively, and anxiety-/depression-like behaviors were measured in the 
EPM, LDT, and MB tasks, as well as TST and FST, on the next day following treatment with the fecal or bacterial suspension.

To understand the ameliorating effects of gut microbiota against depression, the fecal supernatant fraction (10 mg [wet weight] $/ \mathrm{kg} /$ day) of IBD/D+-F or IBD/D-F was orally gavaged into six mice once a day for 5 days. From the next day, the fecal supernatant fraction (10 mg [wet weight] $/ \mathrm{kg} / \mathrm{day}$ ) of HC-F was gavaged once a day for 5 days and anxiety-/depression-like behaviors were measured in the EPM, LDT, and MB tasks, as well as TST and FST, on the next day following treatment with the fecal suspension.

Mice were anesthetized with alfaxalone $(100 \mathrm{mg} / \mathrm{kg}$, intraperitoneal injection: Careside, Gyeonggi-do, Korea). Colons and brains were removed from mice that were transcardially perfused with $4 \%$ paraformaldehyde.

\section{Behavioral tasks}

The EPM task was performed in a plus-maze apparatus (consisting of two open [30 $\times 7 \mathrm{~cm}]$ and two enclosed [30 $\times 7 \mathrm{~cm}]$ arm with 20-cm-high walls extending from a central platform [7 $\times 7 \mathrm{~cm}]$ ), according to the method of Jang et al. [12]. The LDT task was performed in a light/dark box apparatus $(45 \times 25 \times$ $25 \mathrm{~cm}$, consisting of two chambers made of black and white Plexiglass [floor] and polywood [walls] connected by an opening $[7.5 \times 7.5 \mathrm{~cm}]$ located at floor level in the center of the dividing wall), according to the method of Jang et al. [12]. MB task was assessed in a smooth, opaque plastic cage $(30 \times 35 \times 13$ $\mathrm{cm}$ ) with a $5 \mathrm{~cm}$ layer of sawdust and 30 marbles on top of it (five rows or marbles regularly spaced at $2 \mathrm{~cm}$ away from the walls), according to Jang et al. [12]. The number of marbles buried for $30 \mathrm{~min}$ was counted. The TST was performed on the edge of a table, at $30 \mathrm{~cm}$ above it, for $5 \mathrm{~min}$ according to the method of Kim et al. [29]. Mice were judged to be immobile, when they did not move and hung passively. The FST was performed in a round transparent plastic jar $\left(20 \times 40 \mathrm{~cm}^{3}\right)$ containing fresh water $\left(25^{\circ} \mathrm{C}\right)$ to a height of $25 \mathrm{~cm}$ for $5 \mathrm{~min}$, according to Kim et al. [29].

\section{Immunofluorescence assay}

The brains and colons were removed from mice perfused and post-fixed with paraformaldehyde, cytoprotected in $30 \%$ sucrose solution and cryosectioned. Sectioned tissues were immunostained according to the method of Lee et al. [46]. Briefly, the sections were washed with phosphate-buffered saline, blocked with normal serum and incubated with antibodies against NF-kB (p-p65, 1:100, Cell Signaling Technology: cat \# 3033S), LPS (1:100, Abcam: cat \#ab35654), NeuN (1:200, Millipore: cat \#MAB377), Iba1 (1:200, Thermo Fisher Scientific: cat \#PA5-27436), BDNF (1:50, Santa Cruz Biotechnology: cat \# SC-65513), IL-1R (1:100, Abcam: cat \#ab106278) and/or CD11c (1:100, Abcam: cat \#ab11029) overnight, followed by incubation with secondary antibodies conjugated with Alexa Fluor 594 (1:200, Invitrogen) or Alexa Fluor 488 (1:200, Invitrogen) for $2 \mathrm{~h}$. Cell nuclei were stained with 4',6diamidino-2-phenylindole, dilactate (Sigma Aldrich: cat \#F6057). Immunostained sections were observed using a confocal laser microscope. 


\section{Enzyme-linked immunosorbent assay (ELISA) and immunoblotting}

Brain and colon tissues were homogenized with radioimmunoprecipitation assay lysis buffer (Biosesang Inc., Seongnam, Korea: cat \#RC2002) containing a phosphatase inhibitor cocktail and a 1\% protease inhibitor cocktail on ice [29]. For ELISA, the supernatants were transferred into 96-well plates and cytokine levels were determined using ELISA kits (eBioscience, San Diego, CA, USA) [28]. For the assay of corticosterone, IL-1 $\beta$ and IL- 6 levels, the plasma was prepared according to the method of Kim et al. [29] and corticosterone and cytokine concentrations were measured using ELISA kits [12]. For immunoblotting, tissue lysate supernatants were electrophoresed using sodium dodecyl sulfatepolyacrylamide gel electrophoresis and transferred onto a nylon membrane. Proteins were visualized using primary antibody (antibody for claudin-1, claudin-5, or occluding) and then incubated with secondary antibody. Protein bands were visualized using the enhanced chemiluminescence reagent.

\section{Myeloperoxidase activity and limulus amebocyte lysate (LAL) assay}

Myeloperoxidase activity was assayed according to the method of Jang et al. [12]. Fecal and blood endotoxin levels were assayed using an LAL assay kit (Cape Cod Inc., E. Falmouth, MA: cat \# C1500) according to the method of Jang et al. [12].

\section{Pyrosequencing}

Bacterial genomic DNAs were extracted for the fresh feces of HCs, IBD patients, and feces-treated mice using a QIAamp DNA stool mini kit (Qiagen according to the method of Kim et al. [29]. Amplification of genomic DNA was performed using barcoded primers targeted the bacterial 16S rRNA V4 region gene and sequenced using Illumina iSeq 100 (San Diego, CA). ${ }^{28}$ Functional genes was predicted using the phylogenetic investigation of communities by reconstruction of unobserved states (PICRUSt) [39,53]. Linear discriminant analysis (LDA) and cladograms were pictured using the LDA effect size (LefSe) on Galaxy platform (https://huttenhower.sph.harvard.edu/galaxy/) [54]. Pyrosequencing reads were deposited in the short read archive of NCBI under accession number PRJNA666980.

\section{qPCR}

qPCR for gut bacteria was performed on the Rotor-Gene Q® thermocycler using DNA polymerase and SYBR Green I (Takara Bio Inc.: RR820A). PCR amplification reaction was carried out as follows: initial denaturation at $95^{\circ} \mathrm{C}$ for $30 \mathrm{~s}$, followed by 45 cycles of denaturation at $95^{\circ} \mathrm{C}$ for $5 \mathrm{~s}$, annealing at $55^{\circ} \mathrm{C}$ for $30 \mathrm{~min}$, and extension at $72^{\circ} \mathrm{C}$ for $30 \mathrm{~s}$ [28]. Primers for qPCR are indicated in Supplementary Table S2. Each bacterial concentration was calculated relative to 16S rDNA expression using Microsoft Excel.

\section{Statistics}

Experimental data are described as the mean \pm SD using GraphPad Prism 8 (GraphPad Software, Inc., San Diego, CA, USA). Significant differences were analyzed using one or two-tailed Mann-Whitney U test 
for non-parametric test, unpaired $t$ test, ANOVA and Tukey's multiple comparisons test, one-way ANOVA with post-hoc Bonferroni's comparisons test $(p<0.05)$.

\section{Abbreviations}

BDNF: brain-derived neurotrophic factor; CD: Crohn's disease; CFU: colony-forming unit; $\mathrm{D}^{+}$: with depression; $\mathrm{D}^{-}$: without depression; DHL: deoxycholate hydrogen sulfide lactose; ELISA: enzyme-linked immunosorbent assay; EPM: elevated plus maze; FMT: fecal microbiota transplantation; FST: forced swimming test; GAM: general anaerobic medium; HADS-A: hospital anxiety depression scale-anxiety; HADS-D: hospital anxiety depression scale-depression; HC: healthy control; HC-F: healthy control feces; HPA: hypothalamic-pituitary-adrenal; IBD: inflammatory bowel disease; IBD-F: the feces of patients with IBD; LDT: light/dark transition; IL-1: interleukin-1; KoCs: Klebsiella oxytoca with Cronobacter sakazakii; KoEc: Klebsiella oxytoca with Escherichia coli; KoPa: Klebsiella oxytoca with Pediococcus acidilactici; KpBl: Klebsiella pneumoniae with Bifidobacterium longum; KpCs: Klebsiella pneumoniae with Cronobacter sakazakii, KpEc: Klebsiella pneumoniae with Escherichia coli; KpKo: Klebsiella pneumoniae with Klebsiella oxytoca; KpEf: Klebsiella pneumoniae with Enterococcus faecium; KpPa: Klebsiella pneumoniae with Pediococcus acidilactici; LAL: Limulus amoebocyte lysate; LPS: lipopolysaccharide; MB: marble burying; MGB: microbiota-gastrointestinal-brain; NF: nuclear factor; OUT: operational taxonomic unit; qPCR: quantitative real-time polymerase chain reaction; TNBS: 2,4,6trinitrobenzenesulfonic acid; TNF-a: tumor necrosis factor-a; TST: tail suspension test; UC: ulcerative colitis;

\section{Declarations}

\section{Ethics approval and consent to participate}

No applicable

\section{Consent for publication}

Springer Nature remains neutral with regard to jurisdictional claims in published maps and institutional affiliations.

\section{Availability of data and materials}

All the necessary data except pyrosequencing reads are included in the article. Data availability $16 \mathrm{~S}$ sequencing dataset (pyrosequencing reads) was deposited in the NCBI's short read archive under accession number PRJNA666980. Further data will be shared by request.

\section{Competing interests}

The authors declare that they have no conflict of interest. 


\section{Funding}

The present study was supported by the Medical Research Program through the National Research Foundation of Korea (NRF), funded by the Ministry of Science and ICT (NRF- 2017R1A5A2014768).

\section{Acknowledgement}

We thank Dr. Young Joon Oh, a senior scientist in the World Institute of Kimchi, for the technical assistances for the maintenance and usage of the germ-free facility, Dr. Sung-Vin Yim, a professor in the Kyung Hee University Medical Center, for the human stool collection assistance, and Jong-Wook Ryu and Sang-Kap Han for the technical assistance.

\section{Author contributions}

HMJ, JKK, and DHK, conceived and designed experiments. HMJ, JKK, MKJ, YJS, KEL, JWY, CKL, and HJK, performed laboratory experiments or clinical sampling. HMJ, JKK, MKJ, HJK, and DHK, performed data analysis. HMJ, JKK, and DHK, wrote the manuscript.

$\mathrm{HMJ}, \mathrm{JKK}$, and MKJ contributed equally to this work. All authors contributed to reviewing the paper and all authors agreed the present version for submission.

\section{Competing interests}

The authors declare that they have no conflict of interest.

\section{Authors' information (Affiliations)}

Neurobiota Research Center and Department of Life and Nanopharmaceutical Sciences, College of Pharmacy, Kyung Hee University, 26, Kyungheedae-ro, Dongdaemun-gu, Seoul 02447, Republic of Korea Hyo-Min Jang, Jeon-Kyung Kim, Min-Kyung Joo, Yoon-Jung Shin, Kyung-Eon Lee, Jong-Wook Yoo, DongHyun Kim

Department of Internal Medicine, Kyung Hee University School of Medicine, Seoul 02447, Korea Department of Clinical Pharmacology and Therapeutics, College of Medicine, Kyung Hee University, Seoul 02447, Republic of Korea

Chang Kyun Lee, Hyo-Jong Kim

\section{Additional information}

Supplementary information is available for this paper.

\section{References}


1. Ananthakrishnan AN, Bernstein CN, lliopoulos D, Macpherson A, Neurath MF, Ali RAR, Vavricka SR, Fiocchi C. Environmental triggers in IBD: a review of progress and evidence. Nat Rev Gastroenterol Hepatol. 2018;15:39-49.

2. Ananthakrishnan AN. Epidemiology and risk factors for IBD. Nat Rev Gastroenterol Hepatol. 2015;12: 205-217.

3. Zhang YZ, Li YY. Inflammatory bowel disease: pathogenesis. World J Gastroenterol. 2014;20: 91-99.

4. Byrne G, Rosenfeld G, Leung Y, Qian H, Raudzus J, Nunez C, Bressler B. Prevalence of anxiety and depression in patients with inflammatory bowel disease. Can J Gastroenterol Hepatol. 2017;2017: 6496727.

5. Graff LA, Walker JR, Bernstein CN. Depression and anxiety in inflammatory bowel disease: a review of comorbidity and management. Inflamm Bowel Dis. 2009;15: 1105-1118.

6. Neuendorf R, Harding A, Stello N, Hanes D, Wahbeh H. Depression and anxiety in patients with Inflammatory Bowel Disease: A systematic review. J Psychosom Res. 2016;87: 70-80.

7. Bonaz B, Sinniger V, Pellissier S. Anti-inflammatory properties of the vagus nerve: potential therapeutic implications of vagus nerve stimulation. J Physiol. 2016;594: 5781-5790.

8. Mawdsley JE, Rampton DS. Psychological stress in IBD: new insights into pathogenic and therapeutic implications. Gut 2005;54: 1481-1491.

9. Gray MA, Chao CY, Staudacher HM, Kolosky NA, Talley NJ, Holtmann G. Anti-TNFa therapy in IBD alters brain activity reflecting visceral sensory function and cognitive-affective biases. PLoS One. 2018;13: e0193542.

10. Walker JR, Ediger JP, Graff LA, Greenfeld JM, Clara I, Lix L, Rawsthorne P, Miller N, Rogala L, McPhail $\mathrm{CM}$, Bernstein CN. The Manitoba IBD cohort study: a population based study of the prevalence of lifetime and 12-month anxiety and mood disorders. Am J Gastroenterol. 2008;103: 1989-1997.

11. Raison CL, Capuron L, Miller AH. Cytokines sing the blues: inflammation and the pathogenesis of depression. Trends Immunol. 2006;27: 24-31.

12. Mawdsley JE, Macey MG, Feakins RM, Langmead L, Rampton DS. The effect of acute psychologic stress on systemic and rectal mucosal measures of inflammation in ulcerative colitis. Gastroenterology. 2006; 131: 410-419.

13. Jang HM, Lee KE, Lee HJ, Kim DH. Immobilization stress-induced Escherichia coli causes anxiety by inducing NF-KB activation through gut microbiota disturbance. Sci Rep. 2018;8: 13897.

14. Rahimi HR, Shiri M, Razmi A. Antidepressants can treat inflammatory bowel disease through regulation of the nuclear factor-kB/nitric oxide pathway and inhibition of cytokine production: A hypothesis. World J Gastrointest Pharmacol Ther. 2012;3: 83-85.

15. Kamada N, Chen GY, Inohara N, Núñez G. Control of pathogens and pathobionts by the gut microbiota. Nat Immunol. 2013;14: 685-690.

16. Fei N, Bruneau A, Zhang X, Wang R, Wang J, Rabot S, Gérard P, Zhao L. Endotoxin producers overgrowing in human gut microbiota as the causative agents for nonalcoholic fatty liver disease. 
mBio. 2020; 11: e03263-3219.

17. Vasyurenko ZP, Opanasenko LS, Koval GM, Turyanitsa Al, Ruban NM. Cellular and lipopolysaccharide fatty Acid composition of the type strains of Klebsiella pneumoniae, Klebsiella oxytoca, and Klebsiella nonpathogenic species. Mikrobiol Z. 2001;63: 13-21.

18. Jang HM, Lee HJ, Jang SE, Han MJ, Kim DH. Evidence for interplay among antibacterial-induced gut microbiota disturbance, neurolnflammation, and anxiety in mice. Mucosal Immunol. 2018;11:13861397.

19. Ma TY, Iwamoto GK, Hoa NT, Akotia V, Pedram A, Boivin MA, Said HM. TNF-alpha-induced increase in intestinal epithelial tight junction permeability requires NF-kappa B activation. Am J Physiol Gastrointest Liver Physiol. 2004;286: G367-376.

20. Al-Sadi R, Ye D, Boivin M, Guo S, Hashimi M, Ereifej L, Ma TY. Interleukin-6 modulation of intestinal epithelial tight junction permeability is mediated by JNK pathway activation of claudin-2 gene. PLoS One. 2014; 9: e85345.

21. Fuke N, Nagata N, Suganuma H, Ota T. Regulation of gut microbiota and metabolic endotoxemia with dietary factors. Nutrients. 2019; 11: 2277.

22. Jang SE, Lim SM, Jeong JJ, Jang HM, Lee HJ, Han MJ, Kim DH. Gastrointestinal inflammation by gut microbiota disturbance induces memory impairment in mice. Mucosal Immunol. 2018;11:369379.

23. Alam MT, Amos GCA, Murphy ARJ, Murch S, Wellington EMH, Arasaradnam RP. Microbial imbalance in inflammatory bowel disease patients at different taxonomic levels. Gut Pathog. 2020;12: 1.

24. Pittayanon R, Lau JT, Leontiadis GI, Tse F, Yuan Y, Surette M, Moayyedi P. Differences in gut microbiota in patients with vs without inflammatory bowel diseases: A systematic review. Gastroenterology 2020;158: 930-946.

25. Gong D, Gong X, Wang L, Yu X, Dong Q. Involvement of reduced microbial diversity in inflammatory bowel disease. Gastroenterol Res Pract. 2016;2016: 6951091.

26. Lepage P, Häsler R, Spehlmann ME, Rehman A, Zvirbliene A, Begun A, Ott S, Kupcinskas L, Doré J, Raedler A, Schreiber S. Twin study indicates loss of interaction between microbiota and mucosa of patients with ulcerative colitis. Gastroenterology 2011;141:227-236.

27. Weingarden AR, Vaughn BP. Intestinal microbiota, fecal microbiota transplantation, and inflammatory bowel disease. Gut Microbes 2017;8: 238-252.

28. Erben U, Loddenkemper C, Doerfel K, Spieckermann S, Haller D, Heimesaat MM, Zeitz M, Siegmund B, Kühl AA. A guide to histomorphological evaluation of intestinal inflammation in mouse models. Int $J$ Clin Exp Pathol. 2014;7: 4557-4576.

29. Kim JK, Lee KE, Lee SA, Jang HM, Kim DH. Interplay between human gut bacteria Escherichia coli and Lactobacillus mucosae in the occurrence of neuropsychiatric disorders in mice. Front Immunol. 2020;11: 273.

30. Jiang H, Ling Z, Zhang Y, Mao H, Ma Z, Yin Y, Wang W, Tang W, Tan Z, Shi J, Li L, Ruan B. Altered fecal microbiota composition in patients with major depressive disorder. Brain Behav Immun. 
2015;48: 186-194.

31. Collins SM, Surette M, Bercik P. The interplay between the intestinal microbiota and the brain. Nat Rev Microbiol. 2012;10: 735-742.

32. Mayer EA, Savidge T, Shulman RJ. Brain gut microbiome interactions and functional bowel disorders. Gastroenterology. 2014;146: 1500-1512.

33. Cheung SG, Goldenthal AR, Uhlemann AC, Mann JJ, Miller JM, Sublette ME. Systematic review of gut microbiota and major depression. Front Psychiatry. 2019;10: 34.

34. Mastuoka K, Kanai T. The gut microbiota and inflammatory bowel disease. Semin Immunopathol. 2015;37: 47-55.

35. Chang CW, Wong JM, Tung CC, Shih IL, Wang HY, Wei SC. Intestinal stricture in Crohn's disease. Intest Res. 2015;13: 19-26.

36. Zuo T, Ng SC. The gut microbiota in the pathogenesis and therapeutics of inflammatory bowel disease. Front Microbiol. 2018;9: 2247.

37. Kostic AD, Xavier RJ, Gevers D. The microbiome in inflammatory bowel diseases: Current status and the future ahead. Gastroenterology. 2014;146: 1489-1499.

38. Lee KE, Kim JK, Han SK, Lee DY, Lee HJ, Yim SV, Kim DH. The extracellular vesicle of gut microbial Paenalcaligenes hominis is a risk factor for vagus nerve-mediated cognitive impairment. Microbiome 2020;8: 107.

39. Koo JW, Duman RS. Evidence for IL-1 receptor blockade as a therapeutic strategy for the treatment of depression. Curr Opin Invetig Drugs 2009;10: 664-671.

40. Jeong MY, Jang HM, Kim DH. High-fat diet causes psychiatric disorders in mice by increasing Proteobacteria population. Neurosci Lett. 2019;698: 51-57.

41. Jang HM, Han SK, Kim JK, Oh SJ, Jang HB, Kim DH. Lactobacillus sakei alleviates high-fat-dietinduced obesity and anxiety in mice by inducing AMPK activation and SIRT1 expression and inhibiting gut microbiota-mediated NF-KB activation. Mol Nutr Food Res. 2019;63: e1800978.

42. D'IncàR MA. Intestinal permeability in inflammatory bowel disease: Pathogenesis, clinical evaluation, and therapy of leaky gut. Mediators Inflamm. 2015;2015: 628157.

43. Fukui $\mathrm{H}$. Increased intestinal permeability and decreased barrier function: Does it really influence the risk of inflammation? Inflamm Intest Dis. 2016;1:135-145.

44. Farhadi A, Gundlapalli S, Shaikh M, Frantzides C, Harrell L, Kwasny MM, Keshavarzian A. Susceptibility to gut leakiness: a possible mechanism for endotoxaemia in non-alcoholic steatohepatitis. Liver Int. 2008;28: 1026-1033.

45. Laranjo M, Potes ME, Elias M. Role of starter cultures on the safety of fermented meat products. Front Microbiol. 2019;10: 853.

46. Hanchi H, Mottawea W, Sebei K, Hammami R. The genus Enterococcus: Between probiotic potential and safety concerns-An update. Front Microbiol. 2018;9: 1791. 
47. Lewis ZT, Shani G, Masarweh C, Popovic M, Frese SA, Sela DA, Underwood MA, Mills DA. Validating bifidobacterial species and subspecies identity in commercial probiotic products. Pediatr Res. 2016; 79: $445-452$

48. Arboleya S, Watkins C, Stanton C, Ross RP. Gut Bifidobacteria populations in human health and aging. Front Microbiol. 2016;7: 1204.

49. Khan I, Ullah N, Zha L, Bai Y, Khan A, Zhao T, Che T, Zhang C. Alteration of gut microbiota in inflammatory bowel disease (IBD): Cause or consequence? IBD treatment targeting the gut microbiome. Pathogens 2019;8: 126.

50. Barandouzi ZA, Starkweather AR, Henderson WA, Gyamfi A, Cong XS. Altered composition of gut microbiota in depression: A systematic review. Front Psychiatry. 2020;11: 541.

51. Cheung SG. Systematic review of gut microbiota and major depression. Front Psychiatry 2019;10: 34.

52. Lennard-Jones JE. Classification of inflammatory bowel disease. Scand J Gastroenterol. Suppl. 1989;170: 2-6.

53. Langille MG, Zaneveld J, Caporaso JG, McDonald D, Knights D, Reyes JA, Clemente JC, Burkepile DE, Vega Thurber RL, Knight R, Beiko RG, Huttenhower C. Predictive functional profiling of microbial communities using 16S rRNA marker gene sequences. Nat Biotechnol.2013; 31: 814-821.

54. Segata N, Izard J, Waldron L, Gevers D, Miropolsky L, Garrett WS, Huttenhower C. Metagenomic biomarker discovery and explanation. Genome Biol. 2011;12: R60.

\section{Figures}


(a)

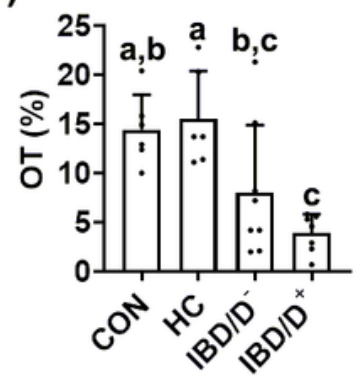

(e)

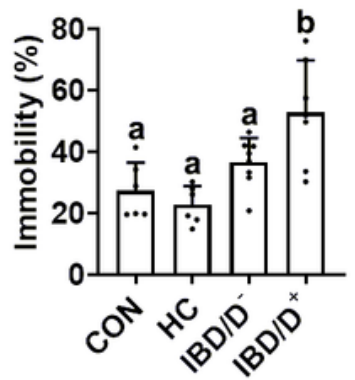

(b)

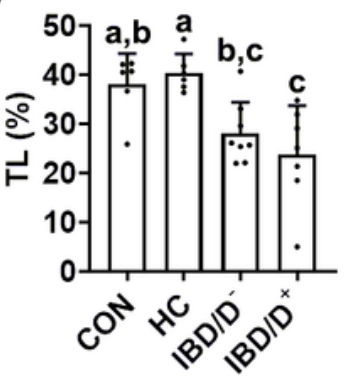

(f)

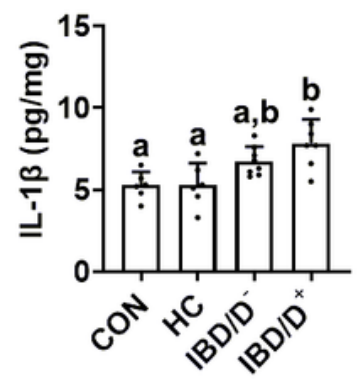

(c)

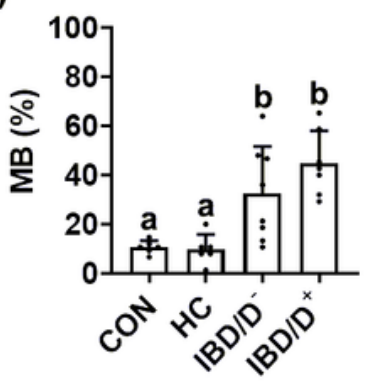

(g)

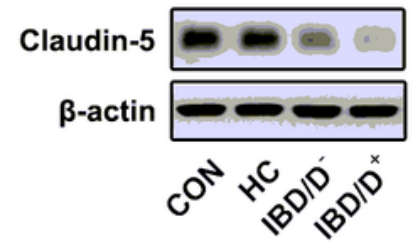

(d)
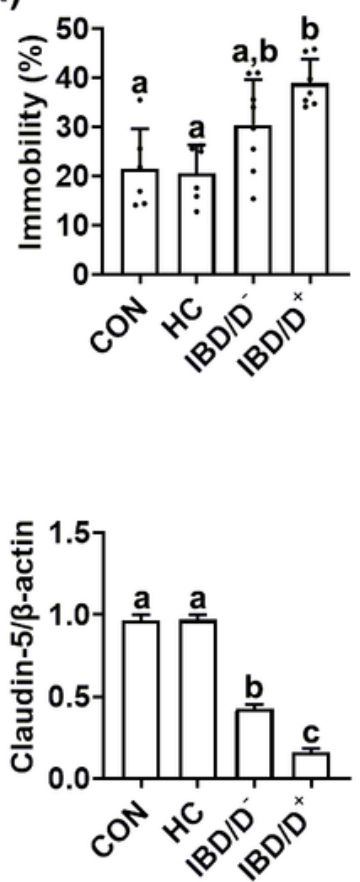

(h)

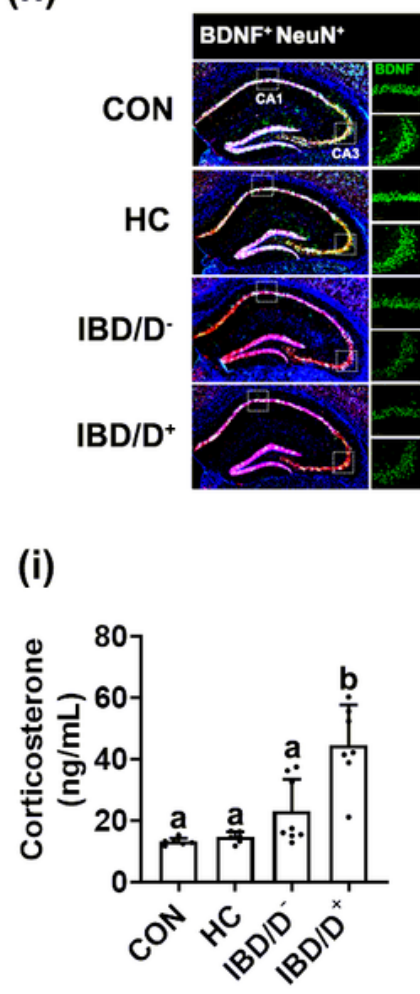

(j)
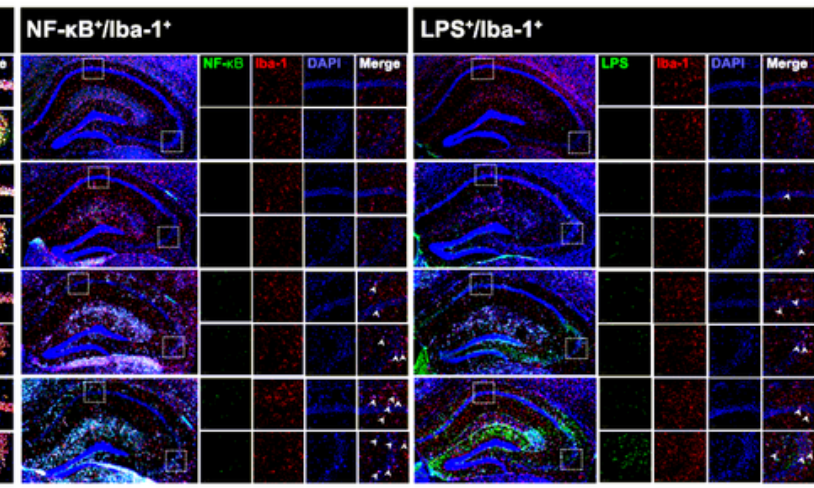

(k)

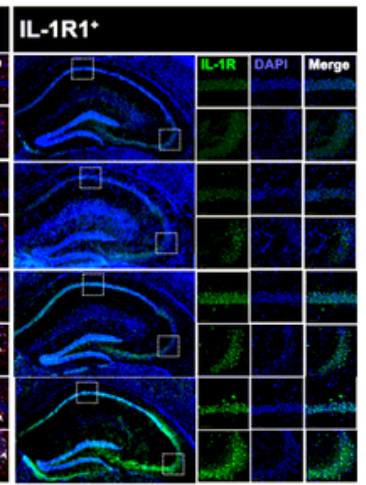

(i)

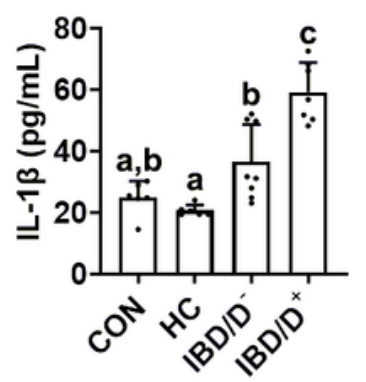

(I)

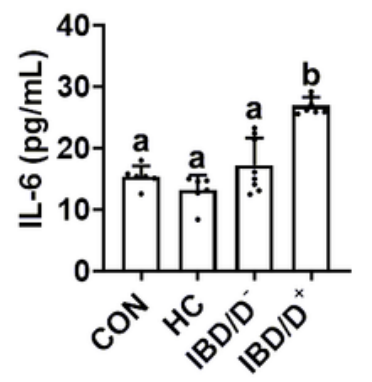

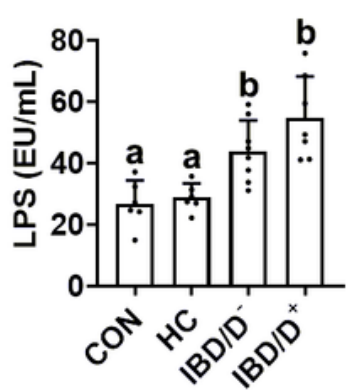

Figure 1

Fecal microbiota transplantation from patients with IBD/DQ or IBD/D+ and healthy individuals $(\mathrm{HC})$ caused anxiety/depression in the transplanted mice. Effects on the occurrence of anxiety/depression in the EPM (a), LDT (b), MB tasks (c), TST (d), and FST (e). (B) Effects on the IL-1 $\beta$ (f), claudin-5 expression (g), BDNF+/NeuN+, NF-kB+//ba1+, LPS+/lba1+ and IL-1R+ cell populations in the hippocampus (h). Effects on the corticosterone (i), IL-1 $\beta$ (j), IL-6 (k), and LPS levels (I). Each HC-F ( $n=6)$, IBD/D®-F (n=8), or 
$\mathrm{IBD} / \mathrm{D}+\mathrm{F}(\mathrm{n}=7)$ was orally transplanted in three mice once a day for 5 days. Control mice were treated with vehicle (saline) instead of fecal suspension. Data values were indicated as mean $\pm S D$ (NC $n=6 ; H C-F$ $n=6 ;|B D / D \otimes n=8 ;| B D / D+n=7$ : each $n$ value is the average obtained from 3 mice). Means with same letters are not significantly different $(p<0.05)$. All data were analyzed using ANOVA with Tukey's multiple comparisons test.

(a)

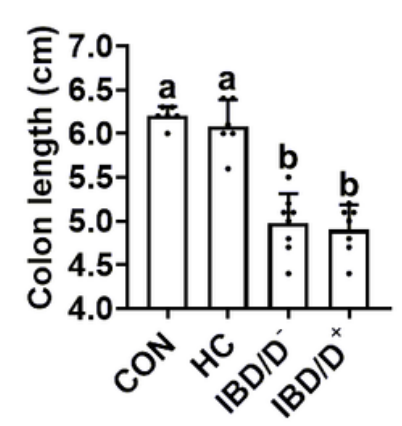

(e)

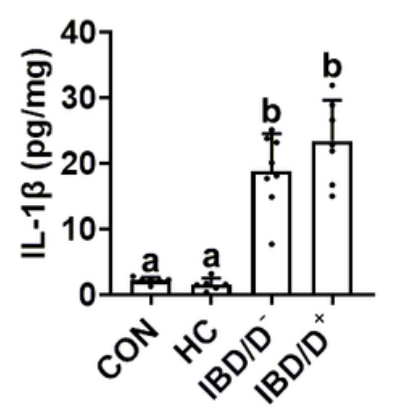

(g)

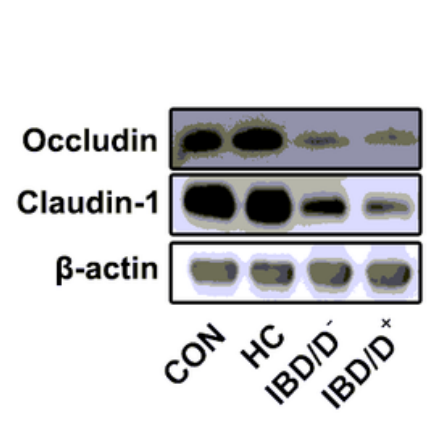

(b)

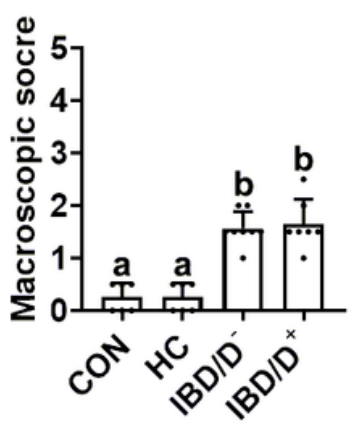

(f)

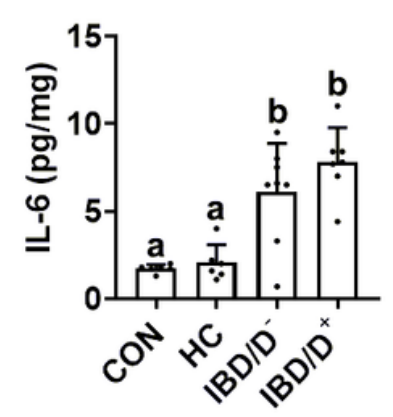

(c)

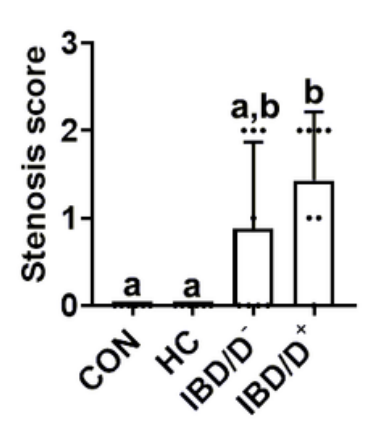

(h)

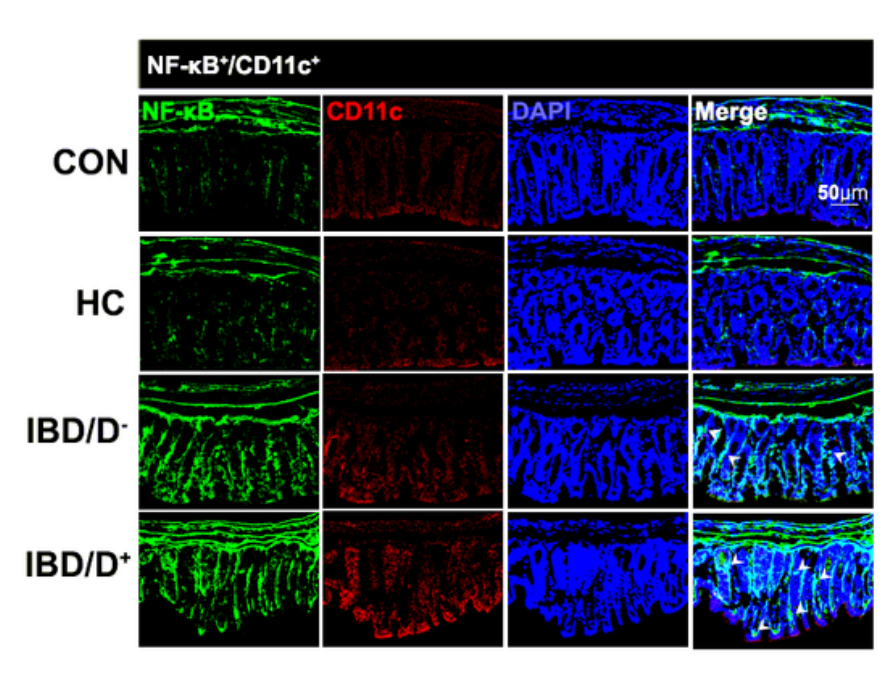

(d)

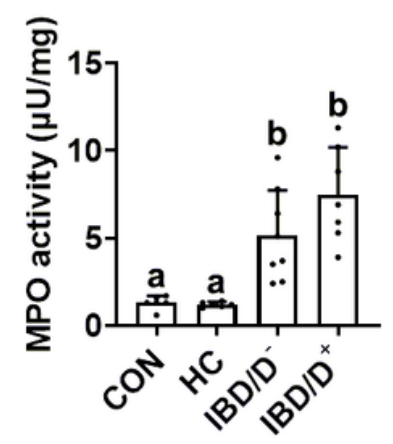

Figure 2

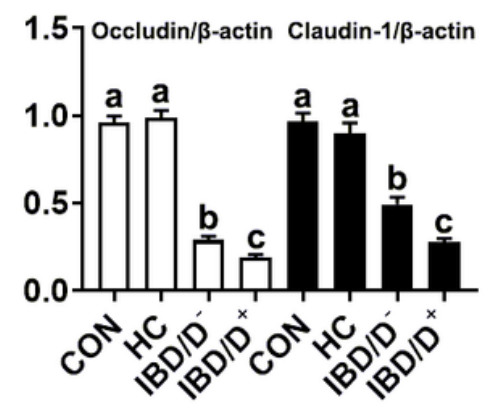


$\mathrm{n}=7$ : each $\mathrm{n}$ value is the average obtained from 3 mice). Means with same letters are not significantly different $(p<0.05)$. All data were analyzed using ANOVA with Tukey's multiple comparisons test.
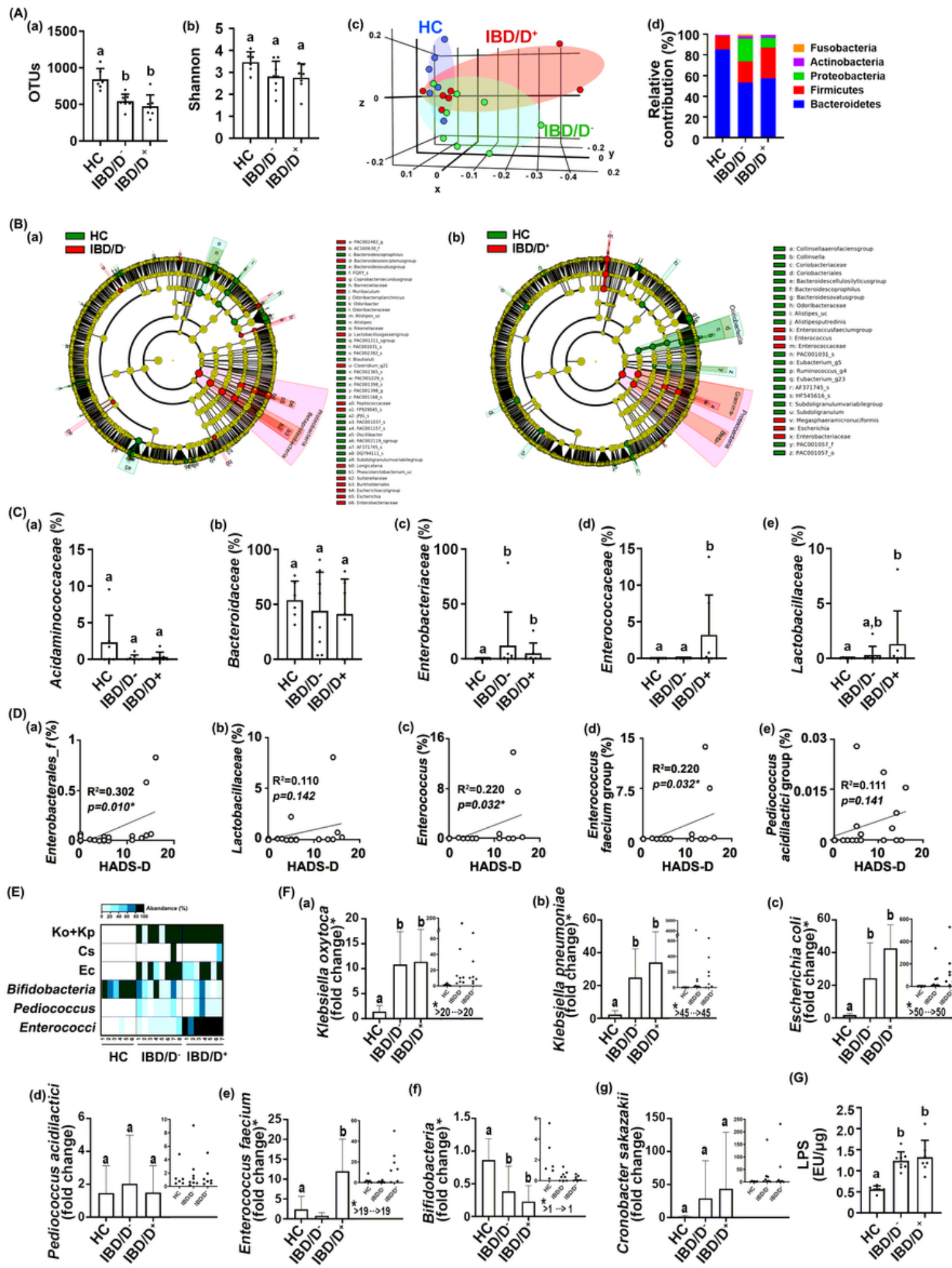

Figure 3

The fecal microbiota composition of patients with IBD/D or IBD/D+ and healthy individuals (HCs). (A) Effects on OTU richness (a), Shannon's index (b), principal coordinate analysis (PCoA) plot based on Jensen-Shannon analysis (c), and phylum level (d). (B) Effects on gut microbiota composition indicated 
by Cladogram between the feces of HCs and IBD/D $\otimes$ patients (a) and between the feces of HCs and IBD/D + patients (b). (C) Effects on the levels of families Acidaminococcaceae (a), Bacteroidaceae (b), Enterobacteriaceae (c), Enterococcaceae (d), and Lactobacillaceae (e). (D) The relationship between the score of HADA-D and the composition of gut bacteria Enterobacterales_f (a), Lactobacillaceae (b), Enterococcus (c), Enterococcus faecium group (d), and Pediococcus acidilactici group (e). The gut microbiota composition was analyzed by using using Illumina iSeq 100. (E) Effects on the levels of gut bacteria Klebsiella oxytoca (Ko)+Klebsiella pneumoniae (Kp), Cronobacter sakazakii (Cs), Escherichia coli (Ec) grown in the DHL agar plate, Bifidobacteria in the BL agar plate, and Pediococcus acidilactici and Enterococci in the En agar plate. (F) Effects on the levels of gut bacteria Klebsiella oxytoca (a), Klebsiella pneumoniae (b), Escherichia coli (c), Pediococcus acidilactici (d), Enterococcus faecium (e), Bifidobacteria (f) and Cronobacter sakazekii (g), assessed by qPCR. (G) Effects on the fecal LPS levels. Data values were indicated as mean $\pm S D(H C n=6 ; I B D / D \otimes n=8 ; I B D / D+n=7)$. Means with same letters are not significantly different $(p<0.05) .(A)(a, b),(C)(b, c, d)$, and $(G)$, Kruskal-Wallis test (nonparametric test); (C)(a) and (F)(a-g), One-way ANOVA Bonferroni's multiple comparisons test (parametric test); (C)(e), Mann Whitney test (nonparametric test). 
(A)

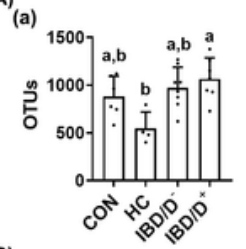

(b)

(B)

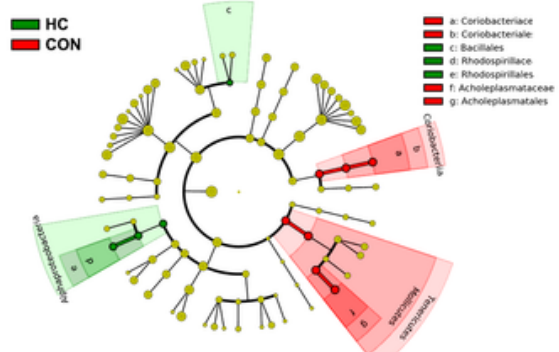

(b)

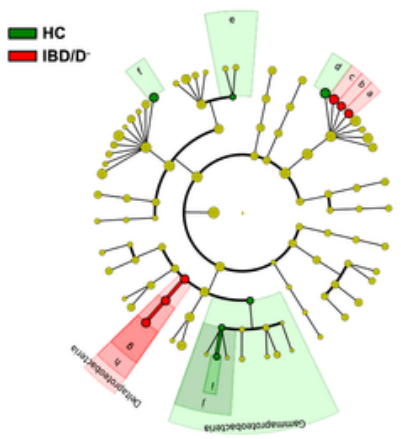

(c)

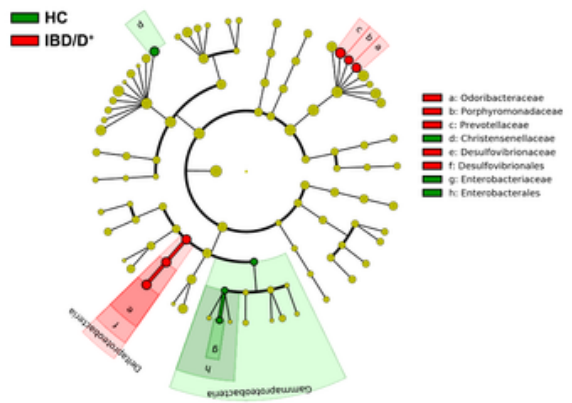

$\left(\mathbb{E}^{(1)}\right)$

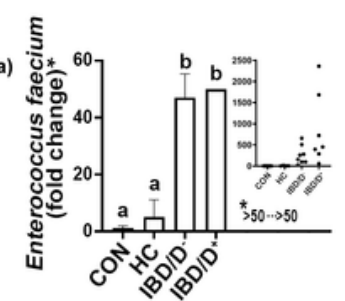

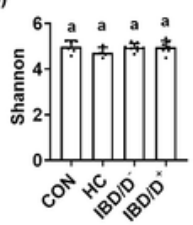

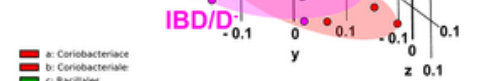

(C)

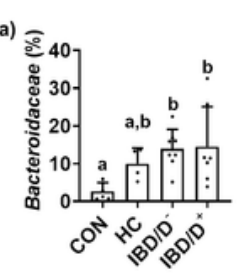

(b)
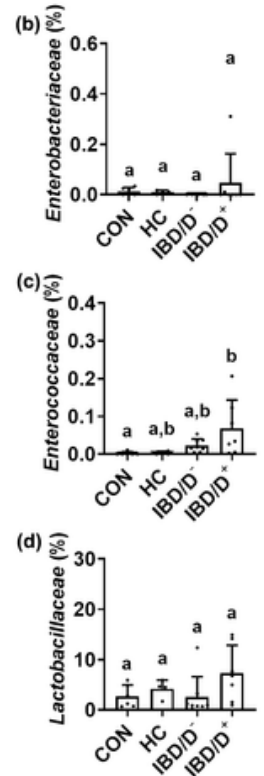

(e)

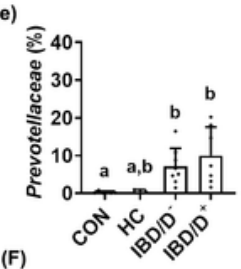

F)

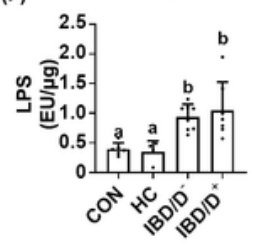

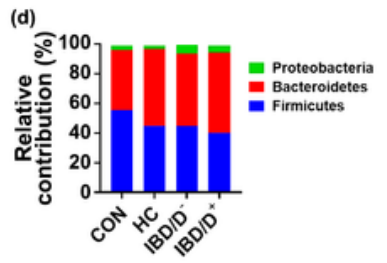

(D)
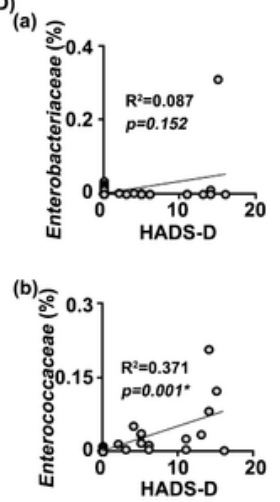

(c)

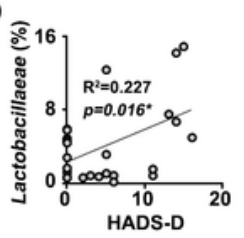

(d)
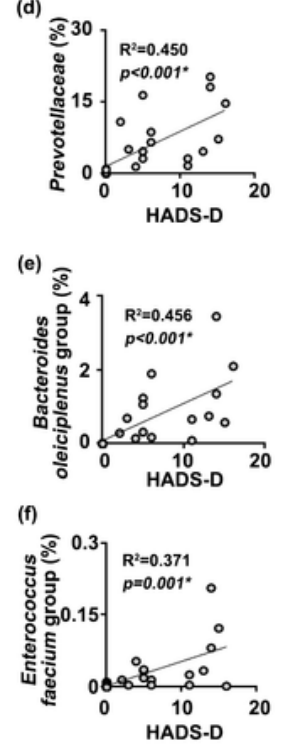

\section{Figure 4}

The fecal microbiota composition of mice orally transplanted with patients with IBD/DQ or IBD/D+ and healthy individuals (HCs). (A) Effects on OTU richness (a), Shannon's index (b), principal coordinate analysis (PCoA) plot based on Jensen-Shannon analysis (c), and phylum level (d). (B) Effects on gut microbiota composition indicated by Cladogram between the feces of norml control mice (CON) and mice transplanted with HC (a), between the feces of HCs and IBD/D® patients (b) and between the feces of HCs 
and IBD/D+ patients (c). (C) Effects on the levels of families Bacteroidaceae (a), Enterobacteriaceae (b), Enterococcaceae (c), Lactobacillaceae (d), and Prevotellaceae (e). (D) The relationship between the score of HADA-D and the composition of gut bacteria Enterobacteriaceae (a), Enterococcaceae (b), Lactobacillaceae (c), Prevotellaceae (d), Bacteroides oleiciplenus (2), Enterococcus faecium group (e). The gut microbiota composition was analyzed by using using Illumina iSeq 100. (E) Effects on the levels of gut bacteria Enterococcus faecium (a) and Pediococcus acidilactici (b), assessed by qPCR. ( $F$ ) Effects on the fecal LPS levels. Data values were indicated as mean $\pm S D(N C n=6 ; H C-F n=4 ; I B D / D \otimes n=8$; IBD/D+ $n=7$ : each $n$ value is the average obtained from 3 mice). Means with same letters are not significantly different $(p<0.05)$. (A) $(a, b),(C)(a, b, d, e)$, and $(F)$, Kruskal-Wallis test (nonparametric test); (B) (c), ANOVA with Tukey's multiple comparisons test; $(E)(a, b)$, One-way ANOVA Bonferroni's multiple comparisons test (parametric test). 
(A)

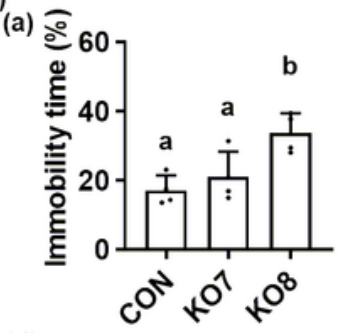

(d)

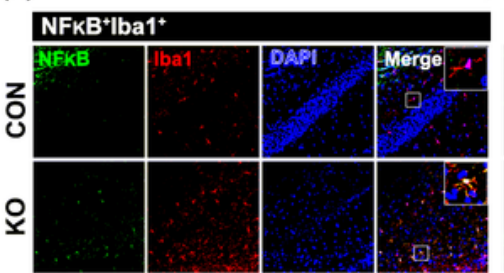

(g)

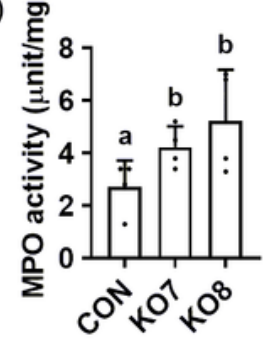

(B)

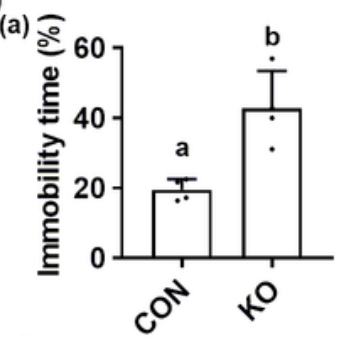

(d)

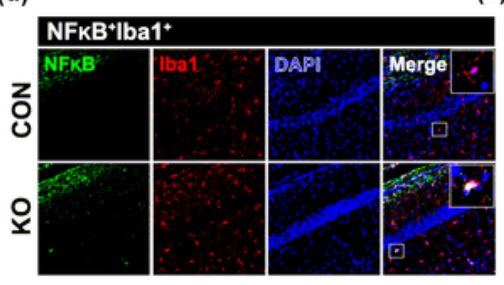

(g)

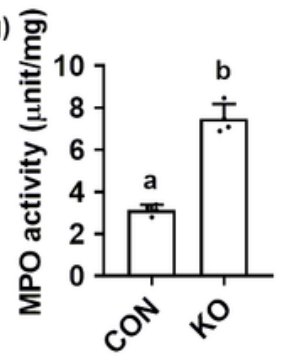

(h)

(b)

(e)

(h)

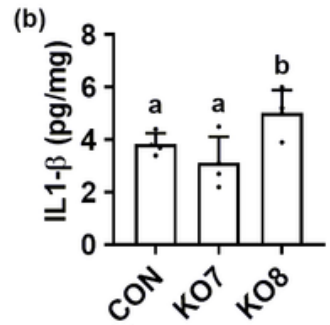

(e)

(c)

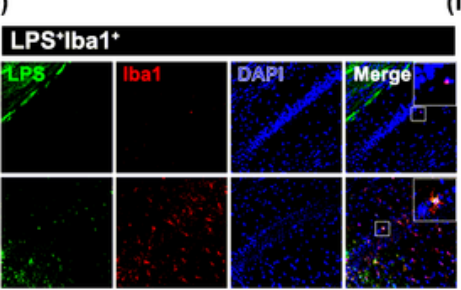

(f)

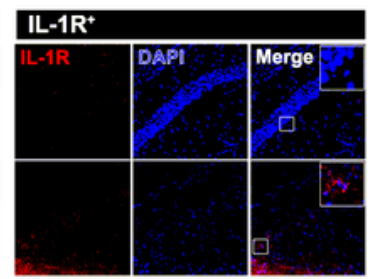

(i)
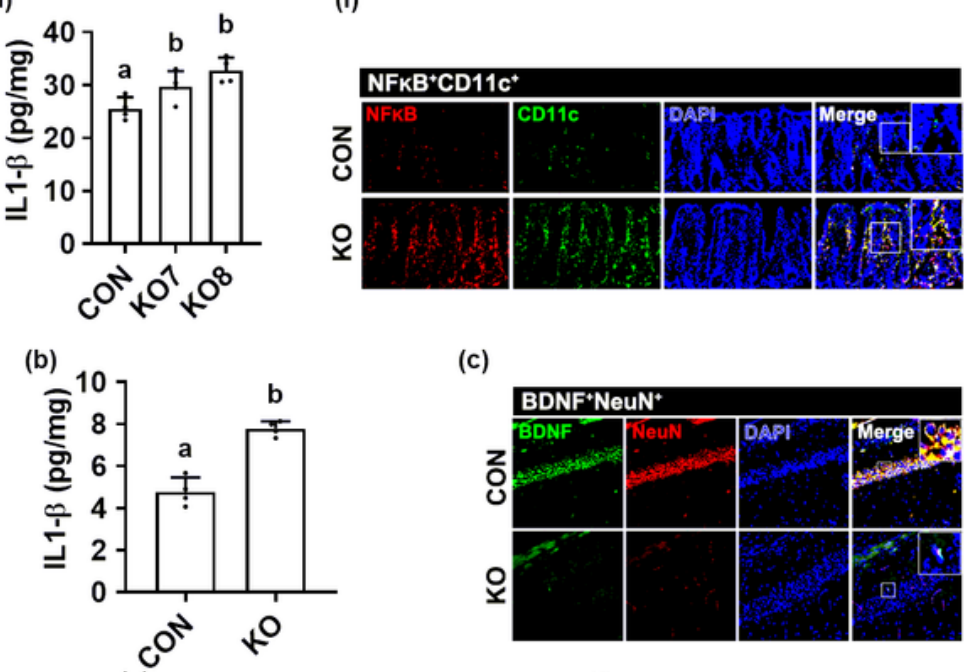

(c)
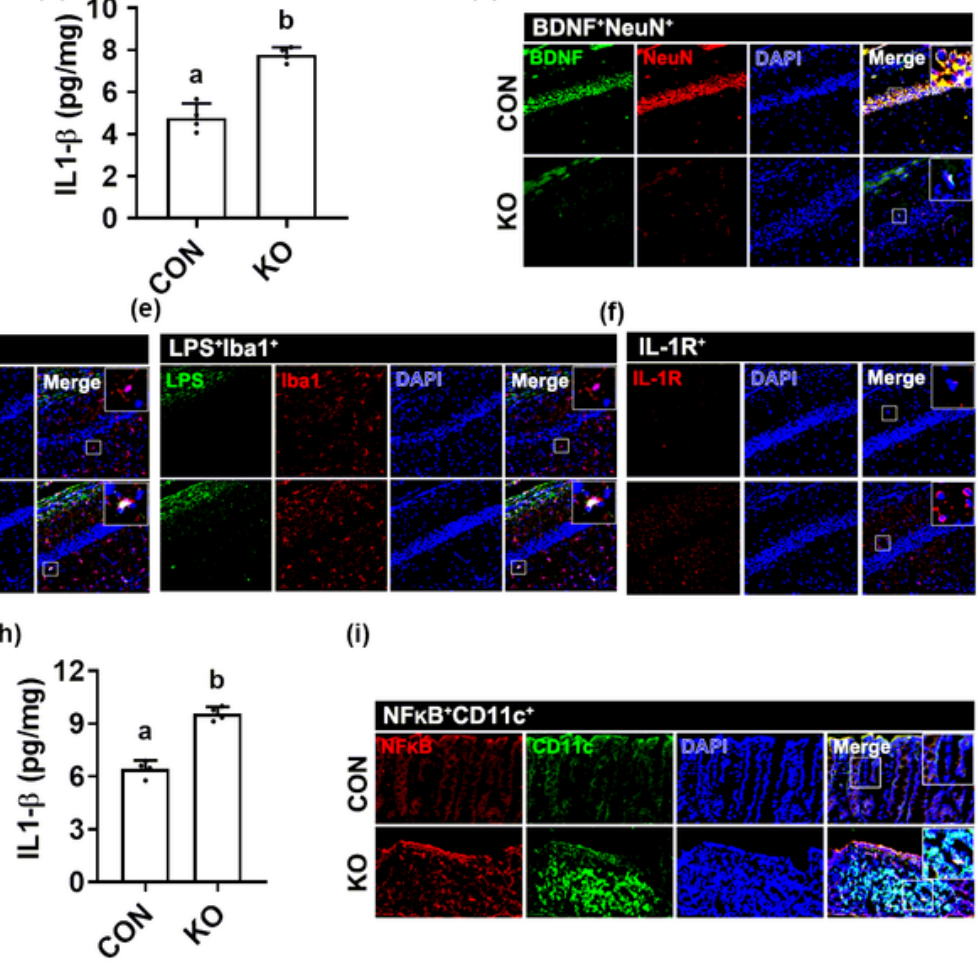

(i)

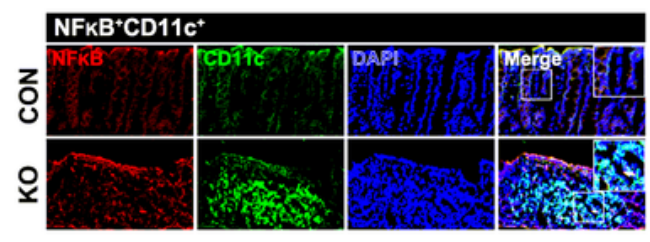

Figure 5

Effect of Klebsiella oxytoca on the occurrence of depression and colitis in germ-free and specificpathogen-free mice. (A) Effect on the occurrence of depression-like behaviors (a) and hippocampal IL-1 $\beta$ level (b), BDNF+/NeuN+ (c), NF-KB+/lba1+ (d), LPS+/Iba1+ (e), and IL-1R+ cell populations (f) in specificpathogen-free mice. Klebsiella oxytoca (KO7, 1×107 CFU/mouse/day; K08, 1×108 CFU/mouse/day) were orally gavaged for 5 days. Control mice (NC) were treated with vehicle (saline) instead of bacterial 
suspension. (B) Effect on the occurrence of depression-like behaviors (a) and hippocampal IL-1 $\beta$ level (b), $\mathrm{BDNF}+/ \mathrm{NeuN}+(\mathrm{c}), \mathrm{NF}-\mathrm{KB}+/ \mathrm{lba} 1+(\mathrm{d}), \mathrm{LPS}+/ \mathrm{lba} 1+(\mathrm{e})$, and IL-1R+ cell populations (f) in germ-free mice. Klebsiella oxytoca (KO, $1 \times 107 \mathrm{CFU} /$ mouse/day) were orally gavaged for 5 days. Control mice (NC) were treated with vehicle (saline) instead of bacterial suspension. Data values were indicated as mean \pm SD ( $n$ $=4)$. Means with same letters are not significantly different $(p<0.05)$. Total unpaired t-test (parametric, one-tailed).

(a)

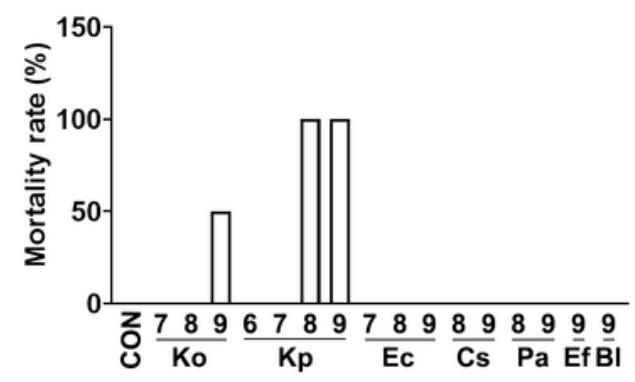

(c)
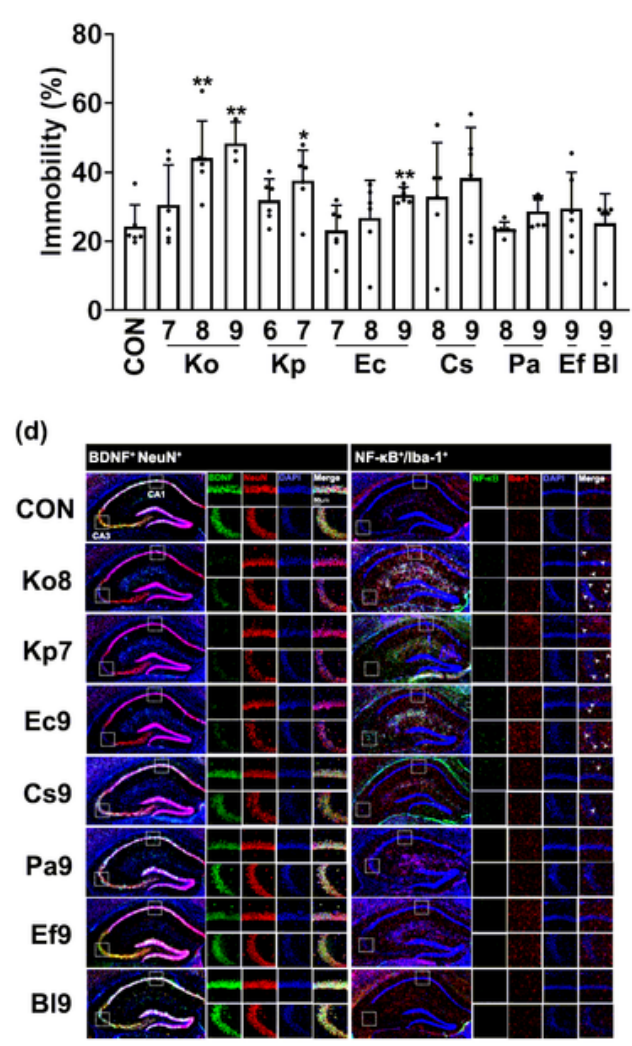

(g)

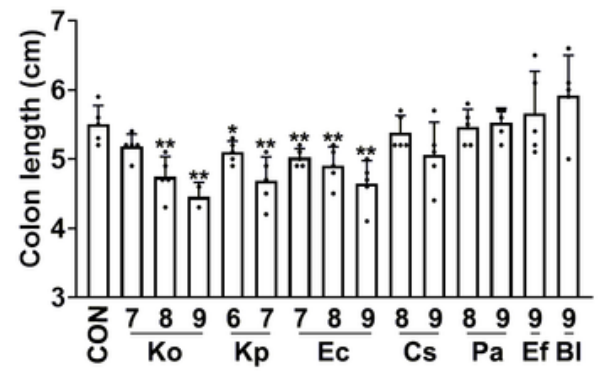

(b)

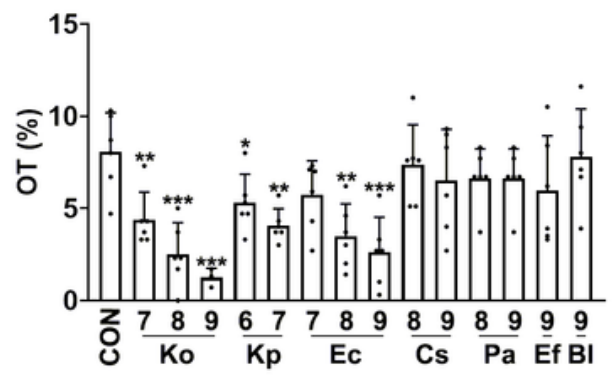

(e)

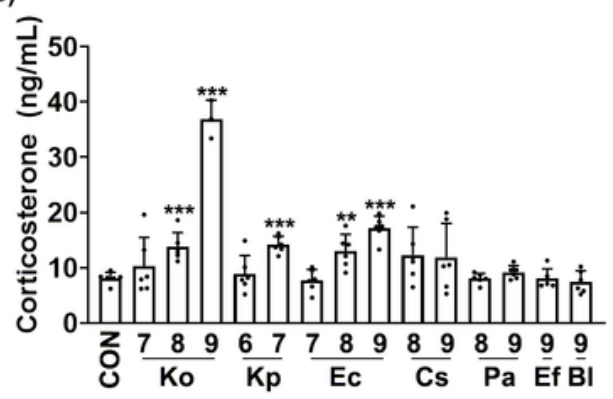

(f)

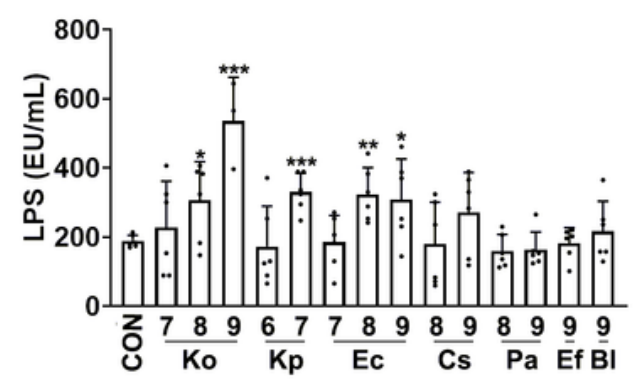

(h)

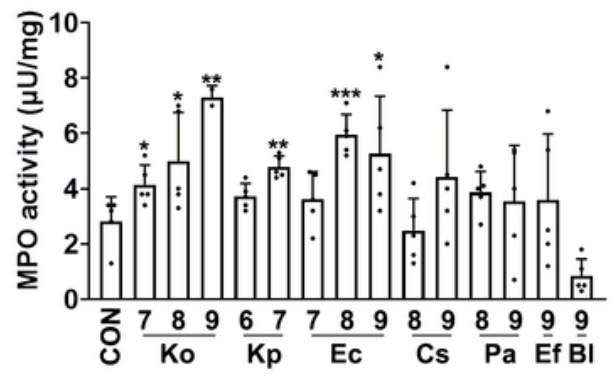

Figure 6 
Effect of Klebsiella oxytoca (Ko), Klebsiella pneumoniae (Kp), Escherichia coli (Ec), Cronobacter sakazakii (Cs), Enterococcus faecium (Ef), Pediococcus acidilactici (Pa), or Bifidobacterium longum (BI) on the occurrence of anxiety/depression and colitis in mice. Effects in the mortality (a). Effects on the time spent in open arms (OT) in the EPM task (b) and immobility time in the TST (c). Effects on the $\mathrm{BDNF}+/ \mathrm{NeuN}+$ and NF-KB+/lba1+ cell population in the hippocampus (d). Effects on the corticosterone (e) and LPS levels (f) in the blood. Effects on the colon length $(\mathrm{g})$ and myeloperoxidase activity $(\mathrm{h})$ in the colon. Each bacterial suspension at doses of $1 \times 106$ [6], $1 \times 107$ [7], $1 \times 108$ [8], $1 \times 109$ [9] CFU/mouse/day) was orally gavaged in six mice once a day for 5 days. Control mice (Con) were treated with vehicle (saline) instead of gut bacterial suspension. Data values were indicated as mean $\pm S D(n=6)$. ${ }^{*}<0.05$ vs Con. ${ }^{* *} p<0.01$ vs Con. ${ }^{* \star *} p<0.001$ vs Con. P Means with same letters are not significantly different $(p<$ $0.05)$. All was analyzed by using unpaired $t$ test. 
(a)

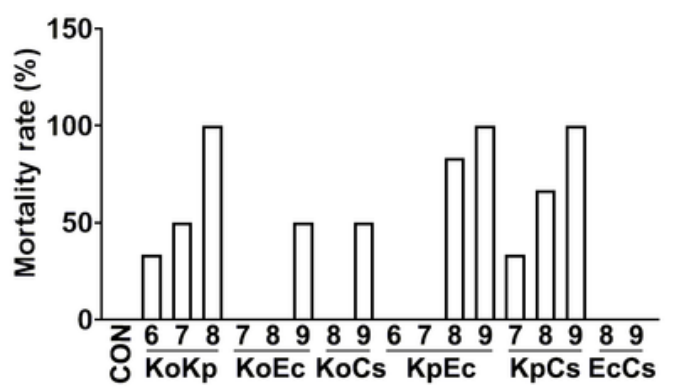

(c)
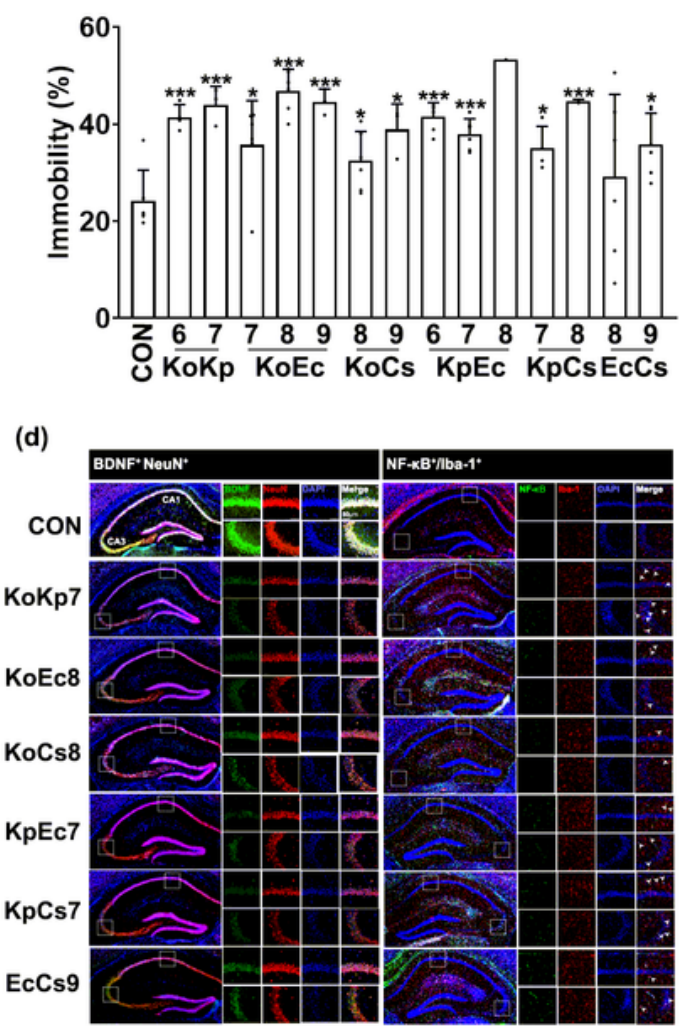

(g)

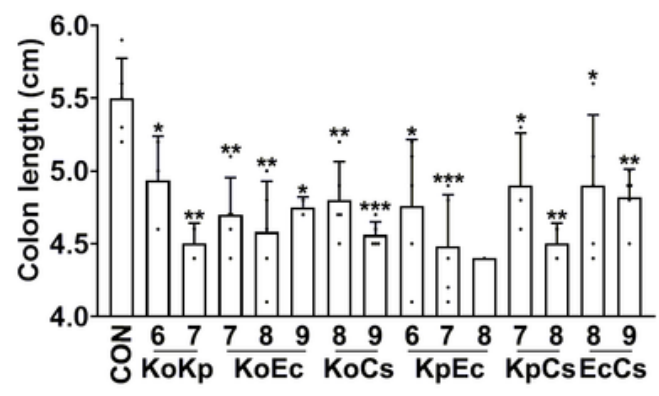

(b)

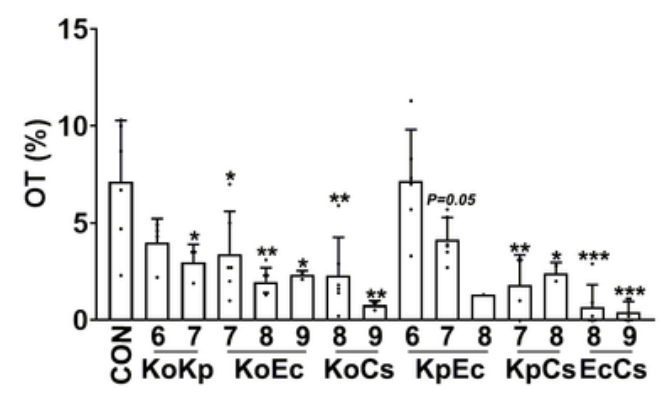

(e)

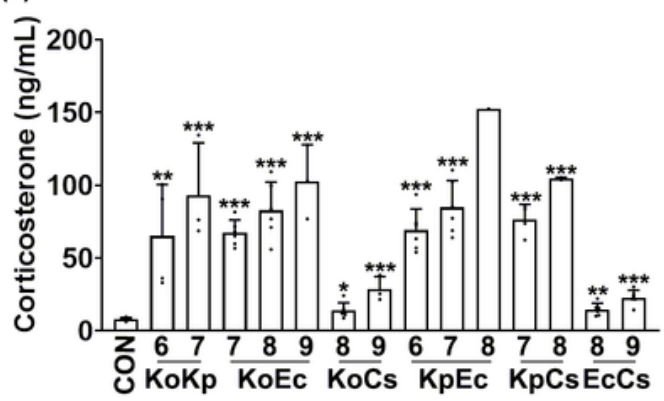

(f)

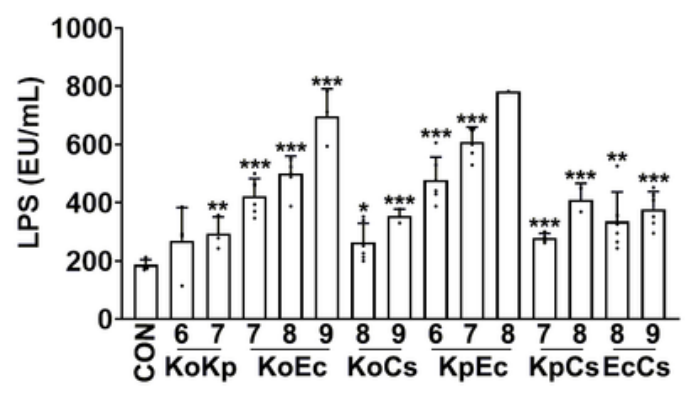

(h)

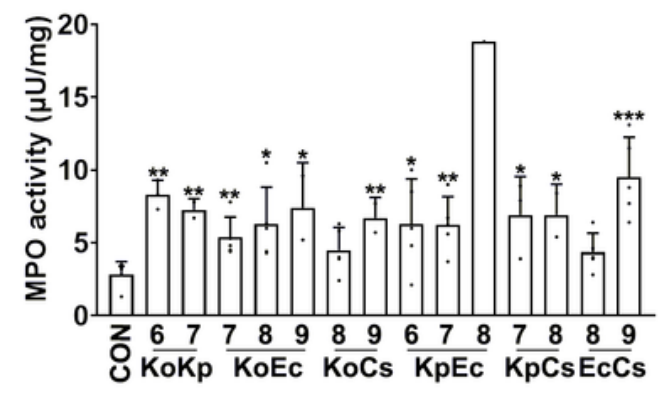

Figure 7

Effects of two bacterial combinations among Klebsiella oxytoca (Ko), Klebsiella pneumoniae (Kp), Escherichia coli (Ec), and Cronobacter sakazakii (Cs) on the occurrence of anxiety/depression and colitis in mice. Effects in the mortality (a). Effects on the occurrence of anxiety/depression in the EPM task (b) and TST (c). Effects on the BDNF+/NeuN+ and NF-KB+/lba1+ cell population in the hippocampus (d). Effects on the corticosterone (e) and LPS levels (f) in the blood. Effects on the colon length (g) and 
myeloperoxidase activity $(\mathrm{h})$ in the colon. Each two bacteria (1:1) combination at doses of $1 \times 106$ [6], $1 \times 107$ [7], $1 \times 108$ [8], 1×109 [9] CFU/mouse/day) was orally gavaged in six mice once a day for 5 days. Control mice were treated with vehicle (saline) instead of gut bacterial suspension. Data values were indicated as mean $\pm S D(n=6) .{ }^{*} p<0.05$ vs Con. ${ }^{* \star} p<0.01$ vs Con. ${ }^{* \star} p<0.001$ vs Con. All was analyzed by using unpaired t test.

(a)

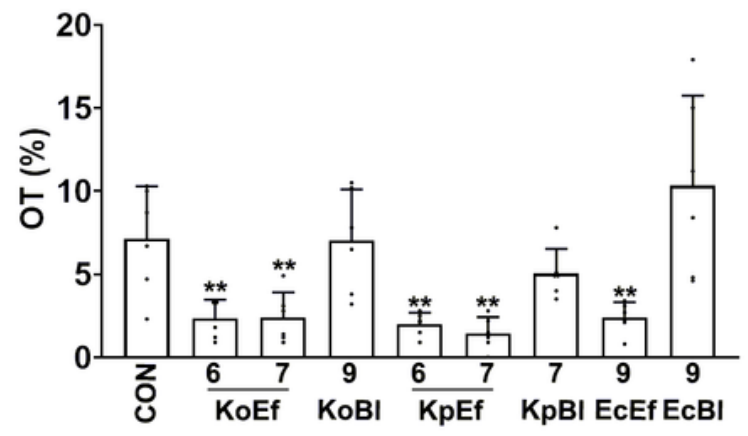

(c)

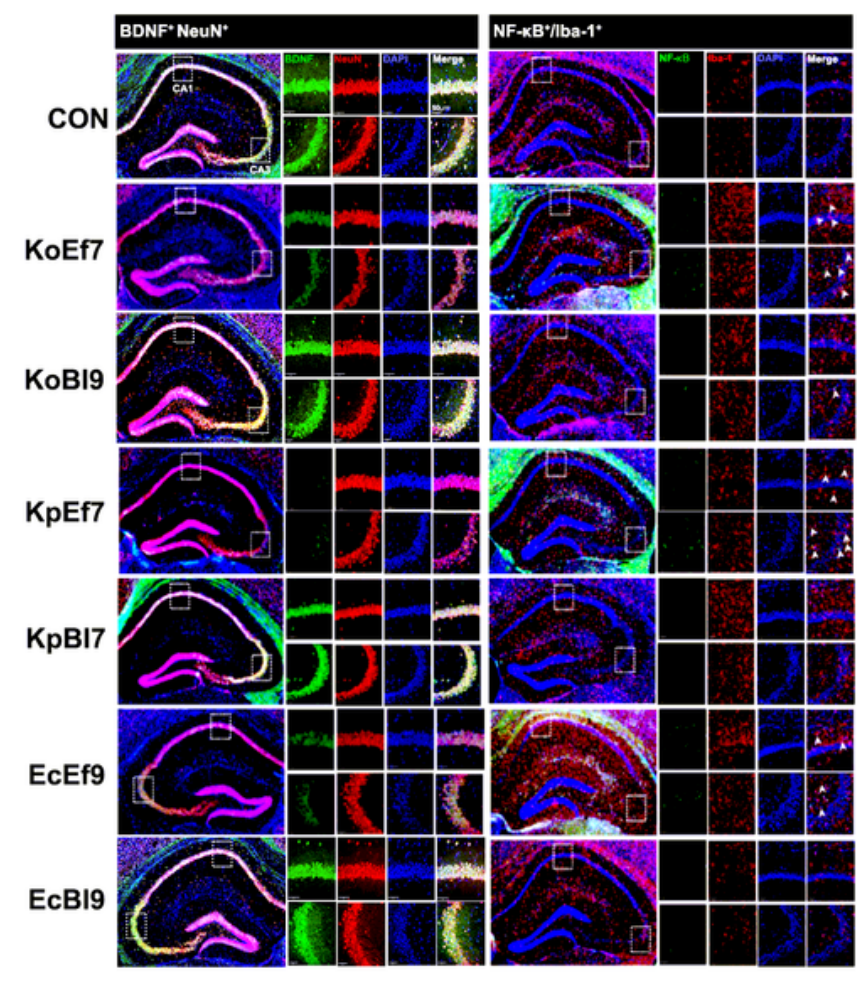

(f)

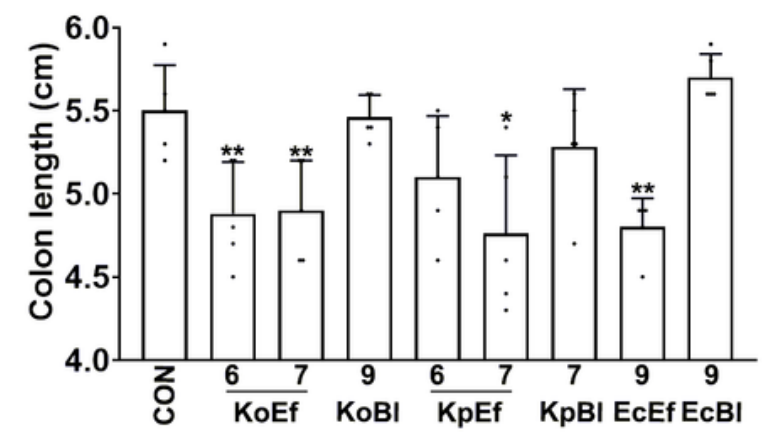

(b)

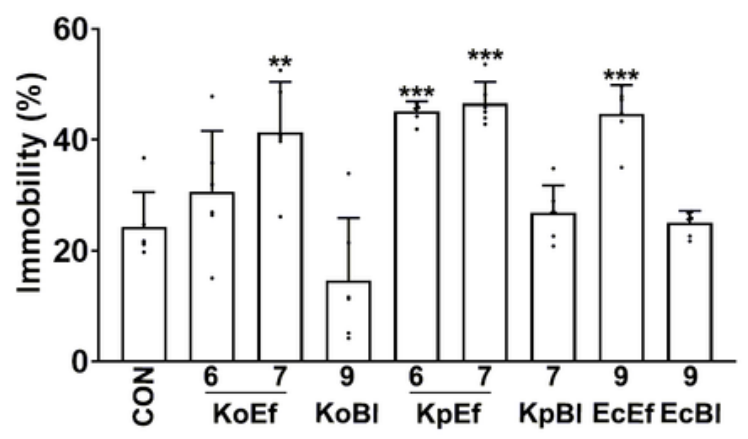

(d)

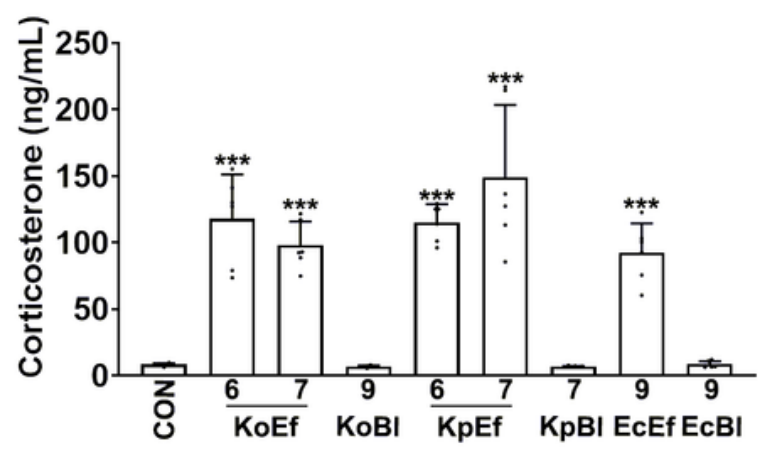

(e)

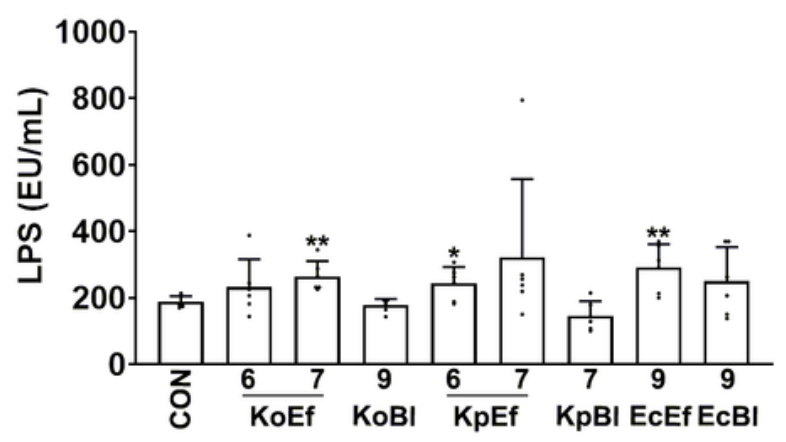

(g)

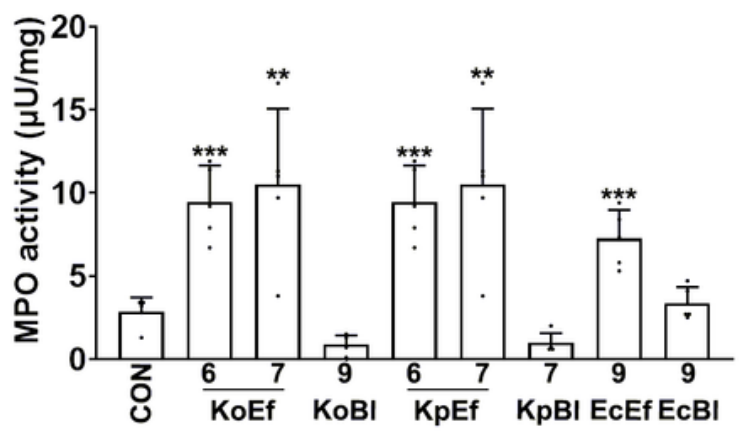

Figure 8 
Combined effects of Enterococcus faecium (Ef) or Bifidobacterium longum (BI) with Enterobacteriaceae $\mathrm{Ko}, \mathrm{Kp}$, or Ec on the occurrence of anxiety/depression and colitis in mice. Effects on the occurrence of anxiety/depression in the EPM task (a) and TST (b). (c) Effects on the BDNF+/NeuN+ and NF-KB+/lba1+ cell population in the hippocampus. Effects on the corticosterone (d) and LPS levels (e) in the blood. Effects on the colon length (f) and myeloperoxidase activity $(g)$ in the colon. Each two bacteria (1:1) combination at doses of $1 \times 106$ [6], $1 \times 107$ [7], $1 \times 108$ [8], 1×109 [9] CFU/mouse/day) was orally gavaged in six mice once a day for 5 days. Control mice were treated with vehicle (saline) instead of gut bacterial suspension. Data values were indicated as mean $\pm S D(n=6) .{ }^{*} p<0.05$ vs Con. ${ }^{* \star} p<0.01$ vs Con. $\star \star \star p<0.001$ vs Con. All was analyzed by using unpaired $t$ test. 
(a)

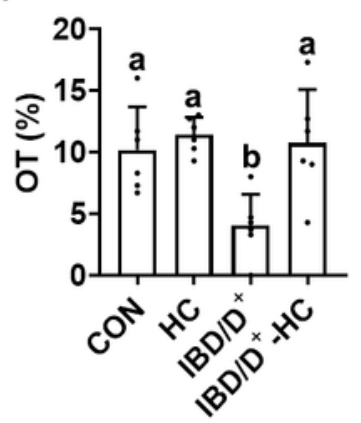

(e)

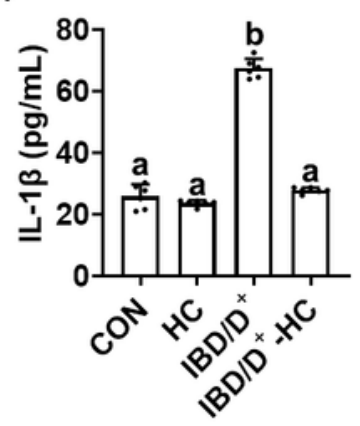

(i)

(B)

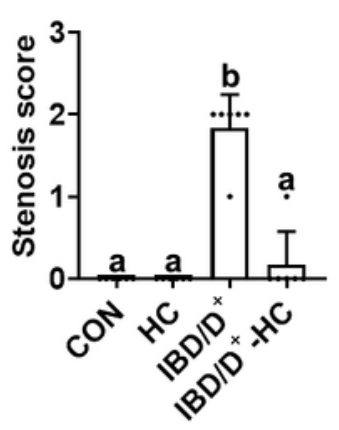

(a)

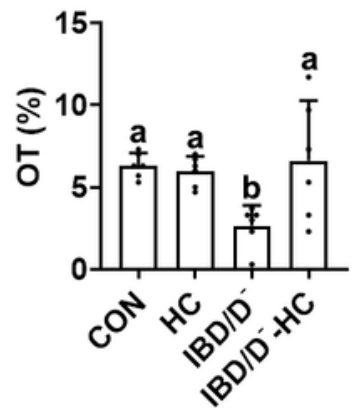

(b)

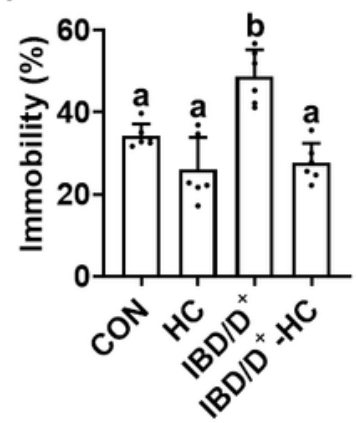

(f)

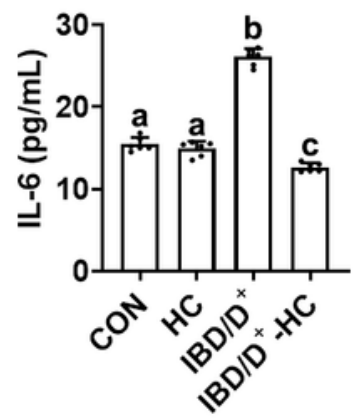

(j)

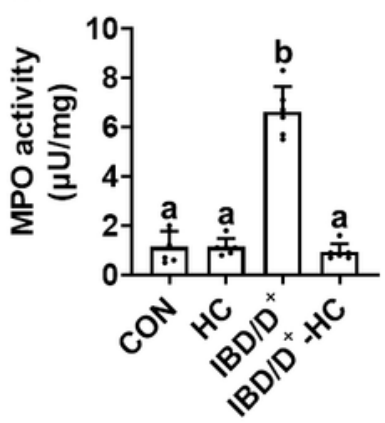

(b)

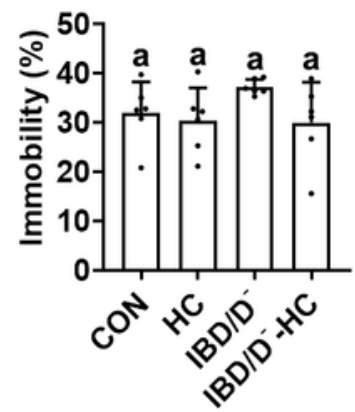

(c)

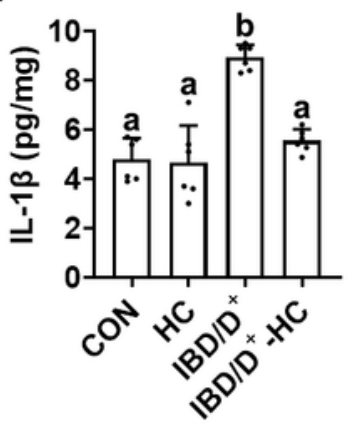

(g)

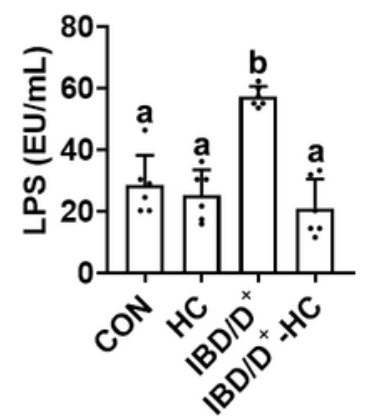

(k)

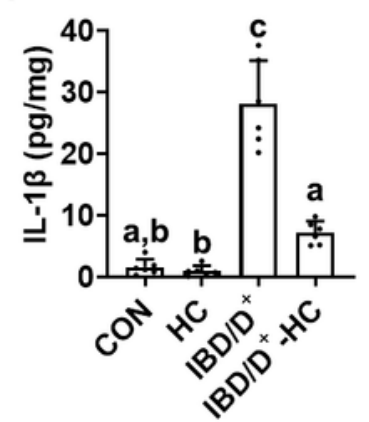

(c)

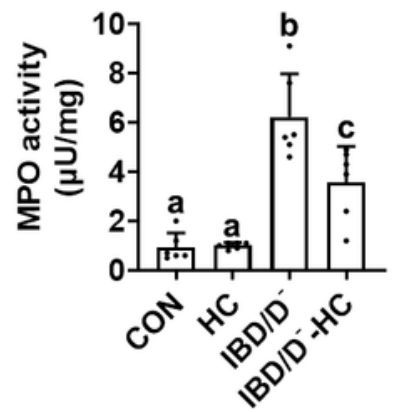

(d)

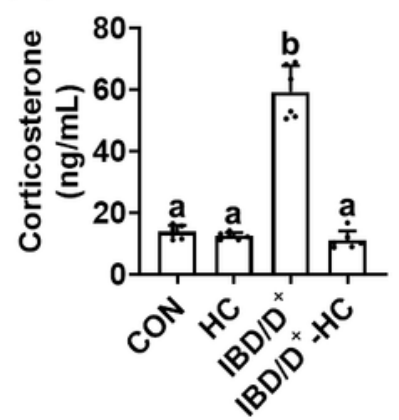

(h)

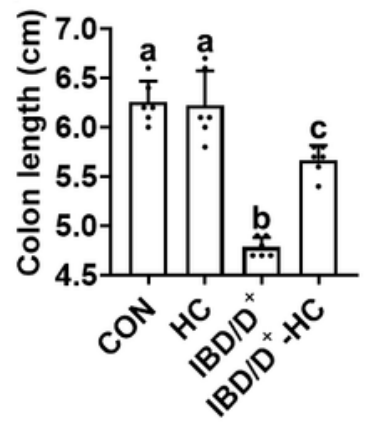

(I)

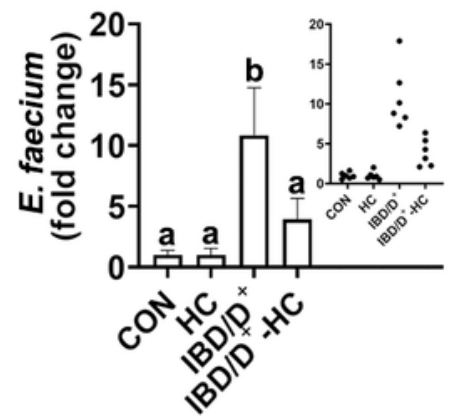

(d)

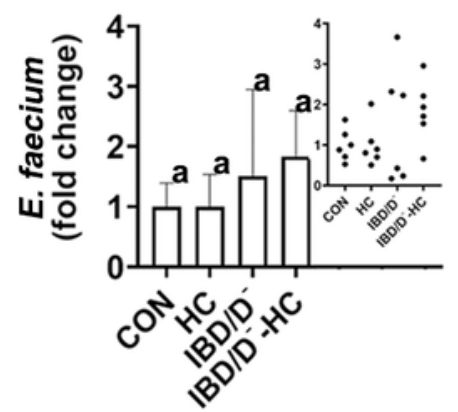

Figure 9

FMT from $\mathrm{HC}$ alleviated IBD/D+-F-induced depression and colitis in the transplanted mice. (A) Effects in IBD/D+-F-gavaged mice. Effects on the occurrence of anxiety/depression in the EPM task (a) and TST (b). Effects on the IL-1 $\beta$ expression (c) in the hippocampus. Effects on the corticosterone (d), IL-1 1 (e), IL$6(\mathrm{f})$, and LPS levels $(\mathrm{g})$ in the blood. Effects on the colon length (h), stenosis score (i), and myeloperoxidase (MPO) activity (j), and IL-1ß expression ( $k$ ) in the colon. (I) Effects on the fecal 
Enterococcus sp. population. (B) Effects in IBD/D囚-F-gavaged mice. (B) Effects in IBD/DQ-F-gavaged mice. Effects on the occurrence of anxiety/depression in the EPM task (a) and TST (b). (c) Effects on MPO activity in the colon. (d) Effects on the fecal Enterococcus sp. population. HF, IBD/D+-F, or IBD/D囚-F suspension was orally transplanted in mice once a day for 5 days. Control mice (Con) were treated with vehicle (saline) instead of fecal suspension. From the next day, HC-F suspension was gavaged in IBD/D+$\mathrm{HC}$ and IBD/DQ-HC mice once a day for 5 days. Con, IBD/DQ, and IBD/D+ mice were treated with vehicle (saline) instead of fecal suspension. Data values were indicated as mean $\pm S D(n=6)$. Fecal Enterococcus sp. population was analyzed by using qPCR. Means with same letters are not significantly different $(p<$ 0.05). All data were analyzed using ANOVA with Tukey's multiple comparisons test.

\section{Supplementary Files}

This is a list of supplementary files associated with this preprint. Click to download.

- MicrobiomeSupplementarylnformation1.docx 